INTERNATIONAL JOURNAL OF APPLIED AND COMPUTATIONAL MATHEMATICS

\author{
ISSN: 2349-5103 (print version) \\ ISSN: 2199-5796 (electronic version) \\ PUBLISHER: SPRINGER
}

ACCEPTED FEBRUARY $1^{\text {ST } 2017}$

\title{
NUMERICAL STUDY OF NONLINEAR HEAT TRANSFER FROM A WAVY SURFACE TO A HIGH PERMEABILITY MEDIUM WITH PSEUDO-SPECTRAL AND SMOOTHED PARTICLE METHODS
}

\author{
O. Anwar Bég * \\ Fluid Mechanics and Propulsion, Mechanical and Aeronautical Engineering, School of Computing, \\ Science and Engineering, UG17 Newton Building, University of Salford, M5 4WT, UK.

\section{S.S. Motsa} \\ School of Mathematics, Statistics and Computer Science, University of KwaZulu-Natal, Private Bag \\ X01, Scottsville 3209, Pietermaritzburg, South Africa. \\ T.A. Bég \\ Renewable Energy and Geodynamics, Israfil House, Dickenson Rd., Manchester, M13, England, UK.
}

A. J. Abbas and A. Kadir

Spray Research Group, Petroleum and Gas Engineering, School of Computing, Science and Engineering, Room G82 Newton Building, University of Salford, M5 4WT, UK.

Ayesha Sohail

Applied Mathematics, Mathematics Department, COMSATS-Lahore, Pakistan.

\begin{abstract}
Motivated by petro-chemical geological systems, we consider the natural convection boundary layer flow from a vertical isothermal wavy surface adjacent to a saturated non-Darcian high permeability porous medium. High permeability is considered to represent geologically sparsely packed porous media. Both Darcian drag and Forchheimer inertial drag terms are included in the velocity boundary layer equation. A high permeability medium is considered. We employ a sinusoidal relation for the wavy surface. Using a set of transformations, the momentum and heat conservation equations are converted from an $(x, y)$ coordinate system to an $(x, \eta)$ dimensionless system. The two-point boundary value problem is then solved numerically with a pseudo-spectral method based on combining the Bellman-Kalaba Quasi Linearization Method with the Chebyschev Spectral collocation technique (SQLM). The SQLM computations are demonstrated to achieve excellent correlation with smoothed particle hydrodynamic (SPH) Lagrangian solutions. We study the effect of Darcy number (Da), Forchheimer number (Fs), amplitude wavelength (A) and Prandtl number (Pr) on the velocity and temperature distributions in the regime. Local Nusselt number is also computed for selected cases. The study finds important applications in petroleum engineering and also energy systems exploiting porous media and undulating (wavy) surface geometry. The SQLM algorithm is shown to be exceptionally robust and achieves fast convergence and excellent accuracy in nonlinear heat transfer simulations.
\end{abstract}

Key words: Natural convection; wave amplitude; porous media; Darcian drag; Forchheimer drag; Nusselt number; Spectral Quasi Linearization Method (SQLM), Smooth Particle Hydrodynamics (SPH); petroleum and geological flows.

\section{*Corresponding Author; email: O.A.Beg@salford.ac.uk}

\section{INTRODUCTION}

The analysis of fluid flow and heat transfer past wavy surfaces and in wavy channels is of fundamental importance in many areas of chemical, petroleum and mechanical engineering. Wavy walls which are 
a form of surface re-structuring (e.g. riblets on internal surfaces in contact with conveyed fluids) have been implemented in various systems in petroleum and energy engineering since reduction of viscous drag in a flow through ducts reduces the pressure losses which in turn decreases costs associated with gas compression [1-3]. Many fundamental studies of flow from wavy surfaces have been communicated. For example, Caponi et al. [4] studied the laminar viscous boundary layer flow over a moving wavy wall.

Another key area of application for wavy surfaces is heat transfer. A number of numerical and experimental studies in this area have been reported. Yao [5] considered the free convection heat transfer past a wavy vertical wall. Moulic and Yao [6] studied the free convection flow past a wavy wall in the presence of uniform heat flux at the wall. Chow and Oosthuizen studied experimentally the natural convection from short cylinders with wavy surfaces. Rees and Pop [7] studied the boundary layer flow and heat transfer on a moving plane numerically. They analyzed the effect of spatially stationary surface waves on the forced convection regime for the case where the Reynolds number, assuming that surface waves have order of unity amplitude and wavelength. Numerical solutions for the local skin friction coefficient and the local Nusselt number for both the cases of a constant wall temperature and a constant wall heat flux were obtained using the Keller box finite difference method. More recently Hossain et al. [8] presented numerical solutions for free convection past a vertical wavy surface with variable viscosity effects. Tashtoush and Abu-Irshaid [9] numerically investigated the heat transfer along a wavy surface with a variable prescribed heat flux. Solutions were obtained for various values of the heat flux exponent, amplitude and Prandtl number. The wavelength of the local Nusselt number and surface temperature variation was shown to be equal to the wavy surface, whereas the wavelength of the average Nusselt number was shown to be half of the wavy surface. Abbassi and Tajik [10] considered the laminar free convection over an inclined wavy surface with constant heat flux using a computational method. Hossain and Islam [11] studied computationally the transient heat transfer in wavy channels. Shalini \& Kumar [12] used the finite element method to analyze the natural convection from a wavy vertical wall to a thermally stratified porous enclosure under non-Darcian assumptions has been analyzed numerically by the finite element method. The effect of inertial forces due to non-Darcian Forchheimer term, thermal stratification level, vertical wavy wall amplitude, wave phase, roughness parameter, and Rayleigh number on the convection process was discussed in detail. The computations revealed multiple circulation zones in flow field, thermal boundary layer development along the lower segment of wavy wall and the occurrence of a "cold region" with large stratification in the temperature field. It was also shown that the Nusselt number could be altered by as much as $90 \%$ by varying the phase of the wavy surface. In the presence of thermal stratification, the influence of non-Darcian effects was shown to be maximized when wave phase of the wavy wall was approximately $300^{\circ}$. Rahman and Badr [13] presented an experimental study of the natural convection heat and mass transfer from a vertical wavy surface in a non-Darcian porous medium Mass-transfer 
coefficients were obtained experimentally for vertical wavy surfaces of varying amplitude-towavelength ratio.

Thus far, to the knowledge of the authors', the free convection heat flow past a sinusoidal wavy vertical surface to a Newtonian fluid in a non-Darcian isotropic porous medium has received limited attention in the literature. This regime is of relevance to petro-chemical (geological) transport phenomena e.g. flows of oils near boundaries of ducts with filters [1-3], solar collectors [14-17] and also petro-chemical materials processing operations [18-20]. In the present study we employ a pseudospectral numerical algorithm which combines the Bellmann-Kalaba quasi-linearization method [21] and the Chebyschev spectral collocation method [22] to obtain accelerated convergence and improved stability for nonlinear boundary value problems. Validation is attained with a smoothed particle hydrodynamic (SPH) [23] code. Details of numerical formulations for both methods employed are described in detail

\section{MATHEMATICAL MODEL}

Consider the steady, laminar free convection flow past a vertical wavy surface embedded in a saturated non-Darcian porous medium. The physical regime is shown in figure $\mathbf{1}$ below. The wavy surface is maintained at a constant wall temperature, $T_{w}$. The porous medium is modeled as an isotropic, homogenous system. For a general 3-dimensional anisotropic medium in a generalized Cartesian coordinate system $(X, Y, Z)$, Coussy [24] has shown that the permeability tensor, $\boldsymbol{K}^{P}$ is second order tensor of the form:

$$
\boldsymbol{K}^{p}=\left[\begin{array}{lll}
K_{X X}^{p} & K^{p}{ }_{X Y} & K^{p}{ }_{X Z} \\
K^{p}{ }_{Y X} & K^{p}{ }_{Y Y} & K^{p}{ }_{Y Z} \\
K^{p}{ }_{Z X} & K^{p}{ }_{Z Y} & K^{p} Z Z
\end{array}\right]
$$

Generally the coefficients are not all unique so that the permeability tensor is symmetric in threedimensional flows and it is therefore assumed that, $K^{p}{ }_{X X}=K^{p}{ }_{Y X}, K^{p}{ }_{Y Z}, K^{p}{ }_{Z Y}$ and $K^{p}{ }_{Z X}=K^{p}{ }_{X Z}$. The permeability coefficients $K_{X X}^{p}, K^{p}{ }_{Y Y}$ and $K^{p}{ }_{Z Z}$ define the permeability in the $\mathrm{X}, \mathrm{Y}$ and $\mathrm{Z}$ directions and therefore only three values are needed to simulate a general three-dimensional anisotropic porous medium. Clearly for the special case of isotropic flow in three dimensions, all three permeabilities will be identical i.e. $K_{X X}^{p}=K_{Y Y}^{p}=K_{Z Z}^{p}=K^{p}$. For two- dimensional porous isotropic flow, as considered in this paper, this simplification still holds and we simulate the hydraulic conductivity of the porous medium with a single permeability, $K^{p}$. Under this assumption, for a Darcy-Forchheimer porous medium, the pressure gradient is related to the porous drag forces, following Vafai and Tien [25], as:

$$
\nabla<\mathrm{P}>=-\frac{\mu}{K^{P}} \mathbf{V}-\frac{b \rho}{K^{P}} \sqrt{\mathbf{V V}} \mathbf{V}
$$


where $P$ is pressure, $\rho$ is density of the non-Newtonian fluid, $\mu$ is the dynamic viscosity of the fluid, $K^{p}$ is the permeability of the porous medium, $\boldsymbol{V}$ is velocity vector and $b$ is the Forchheimer inertial parameter which is related to the geometry of the porous medium. The geometry of the wavy surface is defined by the following relation:

$$
Y=\sigma^{*}\left(X^{*}\right)=a^{*} \sin \left(K^{*} X^{*}\right)
$$

where $\sigma^{*}$ is a geometrical function, $a^{*}$ designates the wavy surface amplitude, $K^{*}$ is the wave number $(=2 \pi / L), L$ is the characteristic length associated with the wavy surface. The surface is therefore arbitrary and specific characteristics can be prescribed by assigning particular values to $a^{*}$ and $K^{*}$, which are appropriate for the heat transfer system being considered. We assume that in the free convection flow, density differences are only incorporated in the buoyancy force i.e. following the Oberbeck-Boussinesq approximation, and implement expressions (2) and (3) above. Additionally viscous dissipation and thermal dispersion effects in the porous medium are ignored as are pressure work effects. Under these approximations, the governing boundary layer equations for the transport of mass, heat and momentum can be shown to take the form:

\section{Mass Conservation}

$$
\frac{\partial U^{*}}{\partial X^{*}}+\frac{\partial V^{*}}{\partial Y^{*}}=0
$$

\section{$X^{*}$-direction Momentum}

$$
\rho\left(U^{*} \frac{\partial U^{*}}{\partial X^{*}}+V^{*} \frac{\partial U^{*}}{\partial Y^{*}}\right)=-\frac{\partial P^{*}}{\partial X^{*}}+\rho g \beta\left(T-T_{\infty}\right)-\frac{\mu}{K^{P}} U^{*}-\frac{\rho b}{K^{P}} U^{* 2}
$$

\section{$Y^{*}$-direction Momentum}

$\rho\left(U^{*} \frac{\partial V^{*}}{\partial X^{*}}+V^{*} \frac{\partial V^{*}}{\partial Y^{*}}\right)=-\frac{\partial P^{*}}{\partial Y^{*}}-\frac{\mu}{K^{P}} V^{*}-\frac{\rho b}{K^{P}} V^{* 2}$

\section{Energy}

$U * \frac{\partial T}{\partial X *}+V * \frac{\partial T}{\partial Y *}=\alpha\left[\frac{\partial^{2} T}{\partial X^{2}}+\frac{\partial^{2} T}{\partial *^{2}}\right]$

where $U^{*}$ and $V^{*}$ are the $X^{*}$ and $Y^{*}$ direction velocities, $\beta$ is the coefficient of volume expansion, $g$ denotes gravitational acceleration, $T_{\infty}$ is the free stream temperature (far from wavy surface), $T$ is temperature in the fluid, $\alpha$ is the thermal diffusivity and all other parameters have been defined previously. The corresponding boundary conditions are prescribed at the wall and in the free stream as follows:

$$
\begin{aligned}
& \text { At } Y^{*}=0: \sigma^{*}(X): U^{*}=V^{*}=0, T=T_{w} \\
& \text { As } Y^{*} \rightarrow \infty: U^{*} \rightarrow 0 ; V^{*} \rightarrow 0 ; T \rightarrow T_{\infty} ; P^{*} \rightarrow P_{\infty}^{*}
\end{aligned}
$$




\section{TRANSFORMATION OF BOUNDARY LAYER EQUATIONS}

We introduce a series of transformations, to eliminate the influence of the wavy surface geometry from the boundary conditions and drop the $Y^{*}$ momentum equation from the analysis. Defining:

$$
\begin{gathered}
X=\frac{X^{*}}{L}, Y=\frac{Y^{*}-\sigma^{*}\left(X^{*}\right)}{L} G r^{\frac{1}{4}}, a=\frac{a^{*}}{L}, U=\frac{U^{*}}{U_{c}}, V=\frac{V^{*}-\sigma^{*}{ }_{X^{*}} U^{*}}{U_{c}} G r^{\frac{1}{4}}, \\
P=\frac{P^{*}}{\rho U_{c}^{2}}, \theta=\frac{T-T_{\infty}}{T_{w}-T_{\infty}}=\frac{T-T_{\infty}}{\Delta T}, \sigma(X)=\frac{\sigma^{*}\left(X^{*}\right)}{L}, \sigma_{X}=\frac{d \sigma}{d X}, U_{c}=(g \beta \Delta T L)^{1 / 2}, \\
G r=\frac{(g \beta \Delta T) L^{3}}{\left[\frac{K^{\prime}}{\rho}\right]^{2}}, \operatorname{Pr}=\frac{v}{\alpha}, D a=\frac{K^{p}}{L^{2}}, F S=\frac{b}{L}
\end{gathered}
$$

where $X$ and $Y$ are dimensionless $X^{*}, Y^{*}$ coordinates, $a$ is dimensionless amplitude, $U$ and $V$ are dimensionless $X, Y$ velocities, $G r$ is the generalized Grashof number, $P$ is dimensionless pressure, $\theta$ is dimensionless temperature function, $\sigma$ is dimensionless wavy surface geometry function, $U_{c}$ is the characteristic velocity, $v$ is dynamic viscosity, $\alpha$ is thermal diffusivity, $\operatorname{Pr}$ is Prandtl number. Implementing eqn. (10) into the boundary layer equations (4) to (7) we arrive at the following nondimensional equations:

$$
\begin{gathered}
\frac{\partial U}{\partial X}+\frac{\partial V}{\partial Y}=0 \\
U \frac{\partial U}{\partial X}+V \frac{\partial U}{\partial Y}=-\frac{\partial P}{\partial X}+\sigma_{X} G r^{\frac{1}{4}} \frac{\partial P}{\partial Y}+\left(1+\sigma_{X}^{2}\right)\left[\frac{\partial^{2} U}{\partial Y^{2}}\right]+\theta-\frac{U}{\operatorname{Re} D a}-\frac{F s}{D a} U^{2} \\
\sigma_{X X} U^{2}+\sigma_{X}\left[U \frac{\partial U}{\partial X}+V \frac{\partial U}{\partial Y}\right]=-G r^{\frac{1}{4}} \frac{\partial P}{\partial Y}+\sigma_{X}\left(1+\sigma_{X}{ }^{2}\right)\left[\frac{\partial^{2} U}{\partial Y^{2}}\right] \\
U \frac{\partial \theta}{\partial X}+V \frac{\partial \theta}{\partial Y}=\frac{1+\sigma_{X}^{2}}{\operatorname{Pr}} \frac{\partial^{2} \theta}{\partial Y^{2}}
\end{gathered}
$$

Proceeding with the analysis, equation (13) is multiplied by $\sigma_{\mathrm{x}}$ and the result added to eqn. (12), a process which eliminates the transverse spatial pressure gradient term, $G r^{\frac{1}{4}} \frac{\partial P}{\partial Y}$ from both equations (12) and (13) and leads eventually to the single ( $X$-direction) momentum equation for the regime: 


$$
\begin{aligned}
U \frac{\partial U}{\partial X}+V \frac{\partial U}{\partial Y}+\left[\frac{\sigma_{X} \sigma_{X X}}{1+\sigma_{X}{ }^{2}}\right] U^{2} & =\left(1+{\sigma_{X}}^{2}\right)\left[\frac{\partial^{2} U}{\partial Y^{2}}\right] \\
& +\frac{\theta}{1+{\sigma_{X}}^{2}}-\frac{U}{\left(1+{\sigma_{X}}^{2}\right) \operatorname{Re} D a}-\frac{F s}{\left(1+{\sigma_{X}}^{2}\right) D a} U^{2}
\end{aligned}
$$

The boundary conditions now become:

$$
\begin{aligned}
& \text { At } Y=0: U=V=0, \theta=1 \\
& \text { As } Y \rightarrow \infty: U \rightarrow 0 ; \theta \rightarrow 0
\end{aligned}
$$

To further simplify the model we introduce now a stream-function transformation. Defining:

$$
\psi=(4 x)^{\frac{3}{4}} F(x, \eta), U=\frac{\partial \psi}{\partial Y}, V=-\frac{\partial \psi}{\partial X}, \eta=\frac{Y}{[4 x]^{\frac{1}{4}}}, \theta=\theta(X, \eta)
$$

The momentum and energy conservation equations therefore reduce to their final forms, as follows:

$$
\begin{aligned}
& \left(1+{\sigma_{X}}^{2}\right) \frac{\partial^{2} F}{\partial \eta^{3}}+3 F \frac{\partial^{2} F}{\partial \eta^{2}}-2\left(1+2 \frac{X \sigma_{X} \sigma_{X X}}{1+\sigma_{X}{ }^{2}}\right)\left[\frac{\partial F}{\partial \eta}\right]^{2}+ \\
& \frac{\theta}{1+{\sigma_{X}}^{2}}-\frac{[4 X]^{1 / 2}}{\left(1+{\sigma_{X}}^{2}\right) \operatorname{Re} D a}\left[\frac{\partial F}{\partial \eta}\right]-\frac{[4 X] F s}{\left(1+\sigma_{X}{ }^{2}\right) D a}\left[\frac{\partial F}{\partial \eta}\right]^{2}=4 X\left[\frac{\partial F}{\partial \eta} \frac{\partial^{2} F}{\partial X \partial \eta}-\frac{\partial^{2} F}{\partial \eta^{2}} \frac{\partial F}{\partial X}\right]
\end{aligned}
$$

$$
\frac{1+\sigma_{X}^{2}}{\operatorname{Pr}} \frac{\partial^{2} \theta}{\partial \eta^{2}}+3 F \frac{\partial \theta}{\partial \eta}=4 X\left[\frac{\partial F}{\partial \eta} \frac{\partial \theta}{\partial x}-\frac{\partial \theta}{\partial \eta} \frac{\partial F}{\partial X}\right]
$$

The corresponding transformed boundary conditions are:

$$
\begin{aligned}
& \text { At } \eta=0: F=\frac{\partial F}{\partial \eta}=0, \theta=1 \\
& \text { As } \eta \rightarrow \infty: \frac{\partial F}{\partial \eta} \rightarrow 0, \theta=0
\end{aligned}
$$

Here $F$ is the reduced stream function, $\frac{\partial F}{\partial \eta}$ is dimensionless velocity and $\eta$ is spanwise pseudosimilarity variable (in $Y$-direction). In due course we shall present a numerical solution for the set of partial differential equations (19) and (20) together with boundary conditions (21) and (22), based on the pseudo-spectral method. For practical engineering problems several parameters are of interest including the local Nusselt number and heat flux at the wall. We shall discuss these later. We first 
describe several important cases of the general model derived which allows a comparative study with previous work. Several important special cases of the flow model can be retrieved. Wavy free convection in a Darcian regime is retrieved for the scenario where heat transfer processes may take place at low filtration velocities so that inertial effects can be ignored. Prescribing $F s=0$ negates the Forchheimer drag in the momentum conservation equation (19). The energy conservation equation (20) is unaffected. For wavy free convection in a pure fluid regime, the regime corresponds to the case of infinite permeability, $K_{p} \rightarrow \infty$ so that the Darcy number, $D a$, becomes infinite. Both porous drag force terms in (19) are therefore removed as the medium now becomes purely viscous Newtonian fluid i.e. the solid matrix particles vanish. A third important case is obtained for free convection from a vertical plate in a Darcy-Forcheimmer porous medium, by setting the wavy surface geometry amplitude to zero, i.e. $a=0$, and therefore $\sigma \rightarrow 0$ i.e. the wavy geometric features are eliminated. This corresponds to the case therefore of a "flat wall".

\section{ENGINEERING FLOW PARAMETERS}

Of considerable interest in materials processing systems is the local Nusselt number, $N u$, which gives a measure of the heat transfer rate at the wavy surface. An appropriate definition for this is:

$$
\frac{N u}{\left[\frac{G r}{4 X}\right]^{\frac{1}{4}}}=\left[1+\sigma_{X}{ }^{2}\right]^{1 / 2}\left[-\frac{\partial \theta}{\partial \eta}(X, 0)\right]
$$

In addition we also define the heat flux, $Q_{w}$, as follows:

$$
Q_{w}=\frac{\kappa \Delta T}{L}\left\{\frac{G r}{4 X}\right\}^{\frac{1}{4}}\left[1+\sigma_{X}^{2}\right]^{1 / 2}\left[-\frac{\partial \theta}{\partial \eta}(X, 0)\right]
$$

For the special case of free convection along a vertical plate in a Darcy-Forchheimer porous medium, amplitude $a \rightarrow 0$ so that $\sigma_{X} \rightarrow 0$ and expression (23) contracts to:

$$
\frac{N u}{\left[\frac{G r}{4 X}\right]^{\frac{1}{4}}}=\left[-\frac{\partial \theta}{\partial \eta}(X, 0)\right]
$$

Similarly the heat flux $\mathrm{Q}_{\mathrm{w}}$ also for the vertical plate case becomes:

$$
Q_{w}=\frac{\kappa \Delta T}{L}\left\{\frac{G r}{4 X}\right\}^{\frac{1}{4}}\left[-\frac{\partial \theta}{\partial \eta}(X, 0)\right]
$$

\section{NUMERICAL SOLUTIONS}

The two-point boundary value problem defined by equations (19) and (20) with conditions (21) and (22) can be solved by a variety of numerical methods including finite elements, generalized differential quadrature, implicit finite difference methods, homotopy analysis, dual recriprocity boundary elements, Lattice Boltzmann techniques etc Here we elect to employ a relatively novel and 
highly optimized, fast convergence procedure, termed the pseudo-spectral method. This is a modification of classical spectral methods and both approaches have been implemented to resolve numerous boundary value problems in multi-physical transport phenomena in recent years. Owens $e t$ al. [26] developed a spectral element technique for non-Newtonian flow from a sphere. Shateyi and Motsa [27] studied radiative heat flux effects on transient stretching flows with a spectral homotopy method. Shateyi and Motsa [28] further analyzed viscosity effects on hydromagnetic convection Sakiadis flow with spectral algorithms. Sibanda et al. [29] derived spectral solutions for viscoelastic channel flow using a Reiner-Rivlin differential fluid model. Bég et al. [30] studied electrical Hartmann and electrical Reynolds number effects on ion drag pumping flows with the Chebyschev spectral collocation method, showing excellent correlation of solutions with network simulation and shooting quadrature. Hoque et al. [31] investigated curved tube hydromagnetic Dean blood flow using spectral collocation and Fourier series, identifying new bracelet vortex patterns. Several studies of micropolar flows with spectral methods have been communicated. Liang et al. [32] used a spectral finite difference algorithm to visualize transient flows of micropolar compressible liquids. They employed a basic Rusanov solver to compute the interface inviscid fluxes and an averaging for viscous fluxes at these interface, and considered steady and unsteady flow from a two-dimensional cylinder and also flow from a flapping wing behind an oscillating cylinder. Recently this procedure has also been implemented to simulate reactive viscoelastic gel propellant flows [33] and also micro-morphic plume dynamics in geosystems [34]. An excellent perspective on spectral techniques is provided by Gottlieb and Orszag [35] with details of convergence addressed by Don and Solomonoff [36]. To solve equations (19)-(20) we initially employ the Bellman-Kalaba quasi-linearization method [37]. The quasilinearisation method (QLM) is essentially a generalized Newton-Raphson Method for solving functional equations. The QLM scheme is derived by linearizing the nonlinear component of the governing equations using Taylor series expansion with the assumption that the difference between the value of the unknown functions at the current iteration level (denoted by $r+1$ ) and the value at the previous iteration level (denoted by $r$ ) is small. Applying the QLM on eqns. $(19,20)$ generates a linearized system of coupled differential equations with variable coefficients and these are then solved iteratively for $r=1 ; 2$ with the Chebyshev pseudo- spectral method. Starting from a given set of initial approximations $F_{0}, \theta_{0}$, the iteration schemes can be solved iteratively for $F_{r+1}(\eta, \xi) ; \theta_{r+1}(\eta, \xi)$ when $r=$ $0 ; 1 ; 2$. We discretize the equation using the Chebyshev spectral collocation method in the $\eta$ (transverse) direction and use an implicit finite difference method in the $\xi$ (streamwise) direction. In this investigation, we apply the spectral collocation method approach described in [27, 28]. Before applying the spectral method, it is convenient to transform the domain on which the governing equation is defined to the interval $[-1,1]$ where the spectral method can be implemented. For the convenience of the numerical computations, the semi-infinite domain in the space direction is approximated by the truncated domain $[0 ; L]$, where $L$ is a finite number selected to be large enough to 
represent the behaviour of the flow properties when $\eta$ is very large. We use the transformation $\eta=$ $L(Y+1) / 2$ to map the interval $[0 ; L]$ to $[-1,1]$. The basic idea behind the spectral collocation method is the introduction of a differentiation matrix $\boldsymbol{D}$ which is used to approximate the derivatives of the unknown variables $F(\eta)$ at the collocation points (grid points) as the matrix vector product:

$$
\begin{aligned}
& \frac{d F^{(1)}}{d \eta}=\sum_{k=0}^{N x} \mathbf{D}_{j_{k}} f\left(Y_{k}\right)=\mathrm{DF}, j=0,1, \ldots \ldots N_{x} \\
& \mathbf{F}=\left[F\left(Y_{0}\right), F\left(Y_{1}\right) \ldots \ldots . . F\left(Y_{N_{x}}\right)\right]^{T}
\end{aligned}
$$

Here $N_{x}+1$ is the number of collocation points and $\boldsymbol{F}$ is the vector function at the collocation points. A similar vector function corresponding to $\theta$ is denoted by $\Theta$, respectively. Higher order derivatives are obtained as powers of $\boldsymbol{D}$, that is:

$F^{(p)}=\mathbf{D}^{p} \mathbf{F}, G^{(p)}=\mathbf{D}^{p} \mathbf{G}, \theta^{(p)}=\mathbf{D}^{p} \Theta$

where $p$ is the order of the derivative. The matrix $\mathbf{D}$ is of size $\left(N_{x}+1\right) \mathrm{X}\left(N_{x}+1\right)$. The grid points on $(\xi, \eta)$ are defined as:

$$
\begin{array}{r}
Y_{j}=\cos \frac{\pi j}{N_{x}}, \xi^{n}=n \Delta \xi, j=0,1 \ldots \ldots . N_{x} \\
n=0,1, \ldots ., N_{t}
\end{array}
$$

where $N_{x}+1, N_{t}+1$ are the total number of grid points in the $\eta$-, $\xi$-directions respectively, and $\Delta \xi$ is the spacing in the $\xi$-direction. The finite difference scheme is applied with centering about a mid-point halfway between $\xi^{n+1}$ and $\xi^{2}$. This mid-point is defined as $\xi^{n+1 / 2}=\left(\xi^{n+1}+\xi^{n}\right) / 2$. The derivatives with respect to $\eta$ are defined in terms of the Chebyshev differentiation matrices. Applying the centering about $\xi^{n+1 / 2}$ to any function, e.g. stream function, $F(\eta, \xi)$ and its associated derivative we obtain:

$$
\begin{aligned}
& F\left(\eta_{j}, \xi^{n+1 / 2}\right)=F_{J}^{n+1 / 2}=\frac{F_{j}^{n+1}+F_{j}^{n}}{2}, \\
& \left(\frac{\partial F}{\partial \xi}\right)^{n+1 / 2}=\frac{F_{j}^{n+1}-F_{j}^{n}}{\Delta \xi}
\end{aligned}
$$

Thus, applying the spectral method with the finite differences in $\xi$ yields: 
$\left[\begin{array}{ll}A_{11} & A_{12} \\ A_{21} & A_{22}\end{array}\right]\left[\begin{array}{l}F_{r+1}^{n+1} \\ \Theta_{r+1}^{n+1}\end{array}\right]=\left[\begin{array}{ll}B_{11} & B_{12} \\ B_{21} & B_{22}\end{array}\right]\left[\begin{array}{l}F_{r+1}^{n} \\ \Theta_{r+1}^{n}\end{array}\right]+\left[\begin{array}{l}K_{1} \\ \Theta_{2}\end{array}\right]$

where $\boldsymbol{A}_{i j} ; \boldsymbol{B}_{i j} ;(i ; j=1 ; 2)$ and $\boldsymbol{K}_{i}(i=1 ; 2)$ are $\left(N_{x}+1\right) X\left(N_{x}+1\right)$ matrices and $\left(N_{x}+1\right) X 1$ vectors, respectively. In all the spectral-based numerical simulations a finite computational domain of extent $L$ $=30$ was chosen in the $\eta$-direction. Through numerical experimentation, this value was found to give accurate results for all the selected governing physical parameters used in the generation of results. Increasing the value of $\eta$ does not affect the results to a significant extent. The number of collocation points used in the spectral method discretization was $N_{x}=100$ in all cases. We remark that the SQLM algorithm is based on the computation of the value of some quantity, say $\mathrm{F}^{\mathrm{n}+1}{ }_{\mathrm{r}+1}$, at each time step. This is achieved by iterating using the quasilinearization method using a known value at the previous step, $n$, as initial approximation. The iteration calculations are carried until some desired tolerance level, $\varepsilon$, is attained. In this study, the tolerance level was set to be $\varepsilon=10^{-7}$. The tolerance level is defined as the maximum values of the infinity norm of the difference between the values of the calculated quantities, that is:

$\max \left\{\left\|F_{r+1}^{n+1}-F_{r}^{n+1}\right\|_{\infty},\left\|G_{r+1}^{n+1}-G_{r}^{n+1}\right\|_{\infty},\left\|\theta_{r+1}^{n+1}-\theta_{r}^{n+1}\right\|_{\infty}\right\}<\varepsilon$

To ensure accuracy of the results, a sufficiently small step size $\Delta \xi$ was used. The step size was chosen to be small enough such that further reduction did not change the obtained results for the flow properties of physical interest. Further details of the formulations and applications of the spectral method are given in [38].

\section{VALIDATION WITH SMOOTH PARTICLE HYDRODYNAMICS (SPH)}

To verify the accuracy of the SQLM algorithm described above, we also employ a robust SPH algorithm to solve the same boundary value problem defined by eqns. (19-22). SPH is a mesh-less particle-based Lagrangian fluid dynamics simulation technique, in which the fluid flow is represented by a collection of discrete elements or pseudo-particles. It was introduced originally for astrophysical fluid dynamics simulations in the late 1970s [39]. It was then further developed for shock gas dynamics and free surface hydrodynamics problems in the 1980s and 1990s by Monaghan and coworkers [40-43]. It has also been applied to viscous-dominated (low Reynolds) number flows [44], ocean hydrodynamics [45], manufacturing fluid mechanics [46], transient pipe flows [47], porous media diffusion [48] and thermal conduction heat transfer [49]. Very recently SPH has been applied to simulate splashdown problems for spacecraft landing modules [50] and also magnetohydrodynamic porous media thermal convection flows for electro-conductive polymer processing [51]. A good 
review of modern applications is given in [52]. In comparison to the Eulerian-based conventional numerical methods, SPH has several distinct advantages, which make it particularly adaptable to nonlinear heat transfer, porous media flows and other branches of viscous fluid dynamics. Within the SPH formulation, the computational domain is discretised by a finite set of interpolating points (particles) with invariant coordinates in the material frame. Although many modifications of SPH have been made in recent years, the foundation for using this technique in a generalized three-dimensional fluid domain, is the three-dimensional Dirac delta function, $\delta^{3}\left(x_{i j}^{\beta}\right)[52]$ which satisfies the following identity:

$$
f\left(x_{i}^{\beta}\right)=\iiint_{\Omega} f\left(x_{i}^{\beta}\right) \delta^{3}\left(x_{i j}^{\beta}\right) d \Omega
$$

The appropriate two-dimensional form is used in the present study, where $\left\{f\left(x_{i}^{\beta}\right)\right\}$ is the kernel approximation of the scalar field $f\left(x_{i}^{\beta}\right)$ at particle $i$. In $\mathbf{S P H}$ the function values and their derivatives at a specific particle are interpolated from the function values at surrounding particles using the interpolating (smoothing) function and its derivatives, respectively:

$$
\begin{aligned}
& f_{i}=\sum_{j} \frac{m_{j}}{\rho_{j}} f_{j} W\left(\left|r_{i}-r_{j}\right|, h\right. \\
& \nabla_{i} f_{i}=\sum_{j} \frac{m_{j}}{\rho_{j}} f_{j} \nabla_{i} W\left(\left|r_{i}-r_{j}\right|, h\right.
\end{aligned}
$$

where $m$ is the mass, $\rho$ is the density and $W$ is the interpolating (smoothing) function with a continuous derivative $\nabla_{\mathrm{i}} W$. The index $i, j$ respectively, denotes the variables at the particle $i, j$ respectively, and $\nabla_{i}$ denotes a derivative according to $\mathbf{r}_{i}$ which is the position vector. The smoothing function $W$ is defined so that its value monotonically decreases as the distance between particles increases. It has a compact support domain, for which the radius is defined by the smoothing length $h$. The smoothing function is normalised and in the limit case, when the smoothing length goes to zero, the smoothing function becomes the Dirac delta function, defined for the general 3-D fluid domain in eqn. (33). Within this study, we have implemented the well-tested and robust cubic B-spline smoothing function. In the current model, numerical tests were conducted to establish computational spatial dependence on the number of SPH particles used. Typically computations do not exceed tens of seconds on an Octane SG Desk workstation. The tolerance level was also set, as in the SQLM simulations, at $\varepsilon=10^{-7}$. Tables 1-3 depict the comparisons of solutions obtained by both SQLM and SPH for skin friction and wall heat transfer gradient distributions. The correlation is excellent. Confidence in the SQLM simulations is therefore justifiably very high. Evidently, all three tables show that skin friction and wall heat transfer rates are strongly decreased with increasing streamwise distance. In other words, 
with further distance from the boundary layer leading edge, the flow is decelerated and the heat transferred to the wall from the saturated porous medium is also suppressed. Comparing tables 1 and 2, we observe that as Darcy number is reduced for Table 2 (it is one tenth the value in Table 1), the skin friction is also significantly depressed. The flow is therefore decelerated and this is attributable to the increasing Darcian resistance and Forchheimer drag force terms in eqn. (19), both of which are inversely proportional to Darcy number. Physically a lower Darcy number (Table 2) implies less space in the porous medium for the fluid to percolate through and greater solid material resistance. The reduced heat transfer is due to the presence of more fibers which enhances thermal conduction between particles and elevates temperatures in the fluid, drawing heat away from the wavy surface. This leads to the reduction in wall heat transfer gradients. Comparing Table 3 with Table 1, we also observe that skin friction is reduced with larger Forchheimer numbers. The Forchheimer (second order) drag force is directly proportional to $F s$, and this implies that with increasing Fs, there will be a simultaneous enhancement in Forchheimer drag. This results in greater resistance to the flow and a drop in skin friction at the wavy wall.

\section{RESULTS AND DISCUSSION}

The convection flow regime is dictated by 5 parameters: Darcy number $(D a)$, Forchheimer number $(F)$, Reynolds number $(R e)$, surface geometrical parameter ( $\sigma$ and its derivatives- this is studied by varying the amplitude, A) and Prandtl number (Pr). Representative results for the velocity, temperature, skin friction and Nusselt number profiles are illustrated in figures 2-11 below.

Fig. 2 shows the influence of amplitude parameter, $a$, on temperature evolution in the boundary layer.There is a strong increase in temperature, with a rise in $a$ values. The parameter, $a$, directly influences $\sigma_{x}$. Only the latter appears in the transformed boundary value problem, however the relation between $a$ and $\sigma_{X}$, is based on non-dimensionalization of eqn. (3). Greater amplitudes of surface waviness clearly accentuate heat diffusion in the boundary layer. The effect is sustained for a reasonable range of values from the plate surface $(\eta=0)$ into the boundary layer regime. The case corresponding to fig. 2 is highly permeable i.e. sparsely packed porous medium ( $D a=1)$, which has also been studied in the context of solar collector engineering systems. Weak Forchheimer effects are present $(F s=1)$. Temperature field troughs arise in the wavy surface at $x=0.5,1,1.5,2,2.5$ etc. Peaks correspond to $x=0.25,0.75,1.25,1.75$. Similar results for amplitude influence have been reported by Moulic and Yao [6] and Rees and Pop [7], however with no tangible explanation of engineering implications. Recent more detailed simulations by Bég et al. [53] and Abdallah and Zeghmati [54] have shown that at greater amplitude wavelengths, there is a significant increase in thermal boundary layer thickness, and this manifests as a corresponding escalation in temperatures within the boundary layer. The geometrical nature of the plate significantly influences the growth and ultimate nature of thermal (and velocity) boundary layers. The curvature affects the thermal conduction rates from the 
solid material to the adjacent fluid which in turn influences diffusion of thermal energy in the boundary layer. There is a strong advantage therefore to be gained in using corrugated surfaces (wavy plates) for materials processing applications, compared with conventional flat plates or stretching sheets which achieve lower temperatures in the boundary layer in the collecting fluid.

Fig. 3 illustrates the temperature response with an increase in Darcy number. The increase in Darcy number accelerates the flow - however the reduction in solid fibers with increasing Darcy number reduces thermal conduction heat transfer in the regime. The latter results in a decrease in temperatures in the material body of the porous medium, and a corresponding increase in heat transferred to the wavy surface. This is advantageous for certain petroleum transport operations and gas conveyance [13] where high rates of heat transfer are desirable at the surface (plate). Temperatures decay quickly from the wall into the boundary layer free stream. The asymptotic nature of solutions with increasing transverse coordinate confirms the imposition of an adequately large boundary condition at infinity in the SQLM computational simulations.

Fig. 4 depicts the response of temperature field to variation in Forchheimer number. The deceleration in the flow with increasing $F s$ values, generates a decrease in momentum boundary layer thickness which aids in energy diffusion and a thickening in the thermal boundary layer. Temperatures exhibit a monotonic decay from a maximum at the wavy surface (wall) to vanishing values towards the edge of the boundary layer regime (free stream), in accordance with the imposed boundary conditions. Higher velocity flow in the porous medium therefore acts to accentuate temperatures i.e. heats the thermal boundary layer and simultaneously enhances thermal boundary layer thickness. Again the smooth convergence of all solutions to zero in the free stream further testifies to the prescription of an adequately large infinity boundary condition in the SQLM numerical code.

Fig. 5 presents the influence of Prandtl number on the temperature distributions. Temperature is evidently strongly depressed with a rise in Prandtl number from $\operatorname{Pr}=0.7$ (air) through 1, 6.7 (water) to 10, 100 (polymeric fluids). $\operatorname{Pr}$ defines the ratio of the momentum diffusivity to thermal diffusivity. It also expresses the ratio of the product of dynamic viscosity and specific heat capacity to the thermal conductivity of the fluid. $\operatorname{Pr}<1$ physically implies that heat will diffuse faster than momentum in the fluid. For $\operatorname{Pr}=1$ the diffusion rates will be the same for heat and momentum i.e. thermal and velocity boundary layer thicknesses will be equal. For $\operatorname{Pr}>1$ momentum will diffuse faster than heat. Clearly the temperature response transverse to the wall is similar to that reported for flat plate surfaces in classical heat transfer. Temperatures are very effectively suppressed with working fluids with larger Prandtl numbers which are characteristic of petro-chemicals, oils etc [2]. This will also manifest with greater heat transfer rates to the wall, producing optimized performance. As with earlier figures, the distributions converge smoothly to the vanishing values in the free stream, confirming that a sufficiently large infinity boundary condition has been prescribed in the SQLM simulations.

Fig 6. depicts the velocity response to an increase in wave amplitude, a. near the plate surface there is an initial deceleration in the velocity; further into the boundary layer, transverse to the wall, the 
velocity is elevated. This has also been observed by numerous researchers including Rahman and Badr [13] (for Newtonian wavy surface heat transfer in porous media) and also Chiu and Chou [14] for non-Newtonian natural convection flow from wavy surfaces. Hydrodynamic boundary layers are thicker near the crests of the wavy surface than the troughs, as will be elaborated when we consider velocity field variation in the streamwise direction ( $x$-coordinate). The sinusoidal behaviour is not featured in the transverse direction ( $\eta$-coordinate). The enhancement in acceleration of the flow is maximized further from the wavy surface. This has important implications for drag-reduction since lower drag results in lesser pressure losses in actual systems which serve to improve economic efficiency in petroleum pumping operations.

Fig. 7 shows the velocity evolution with a variation in Darcy number. Inspection of the transformed momentum equation (19) demonstrates that the Darcian drag, $-\frac{[4 X]^{1 / 2}}{\left(1+\sigma_{X}{ }^{2}\right) \operatorname{Re} D a}\left[\frac{\partial F}{\partial \eta}\right]$, is inversely proportional to Darcy number (for fixed wavy amplitude and Reynolds number), the latter being directly proportional to the hydraulic conductivity i.e. permeability, of the porous medium. Darcian drag force is therefore inversely proportional to permeability. With increasing $D a$ the porous medium permeability in the channel is progressively elevated. In the limit as $D a \rightarrow \infty$, the porous media fibres disappear and the regime becomes purely viscous fluid. Effectively higher $D a$ values will deplete the Darcian resistance and this will accelerate the flow as observed in fig. 7. There will be an associated thinning in the momentum boundary layer thickness and drag reduction.

Fig. 8 illustrates the distribution of velocity with transverse coordinate and variation in Forchheimer number, Fs. The Fs parameter is associated with the second order Forchheimer resistance term, $-\frac{[4 X] F s}{\left(1+\sigma_{X}{ }^{2}\right) D a}\left[\frac{\partial F}{\partial \eta}\right]^{2}$ in the normalized momentum eqn. (19). Forchheimer drag is directly proportional to the parameter, Fs. An increase in $F s$ markedly decelerates the flow but serves to enhance the temperatures in the boundary layer. Forchheimer effects are associated with higher velocities in porous media transport. Forchheimer drag however is second order and the increase in this "form" drag effectively swamps the momentum development, thereby decelerating the flow and causing slower momentum diffusion. This aids thermal diffusion and accentuates temperatures. The term "non-Darcian" does not allude to a different regime of flow, but to the amplified effects of Forchheimer drag at higher velocities. Momentum boundary layer thickness is elevated with increasing Forchheimer number and this indicates that the net drag (Darcian first order and second order drag) is increased. Forchheimer drag therefore acts in principal in the opposite sense to surface waviness and retards the flow.

Fig. 9 shows the response of velocity field to a variation in Prandtl number. As with temperature field, described earlier, velocity field is strongly depressed with greater Prandtl number. Momentum (hydrodynamic) boundary layer thickness is conversely enhanced due to the deceleration in the flow. 
Prandtl number embodies the relative role of momentum diffusion rate to thermal diffusion rate. For $\operatorname{Pr}>1$, momentum duffuses faster than heat whereas for $\operatorname{Pr}<1$ heat diffuses faster than momentum. Whereas temperatures decay consistently from the wavy wall into the free stream, velocity initially ascends from the wavy surface and achieves a maximum some distance into the boundary layer. Thereafter velocity profiles all decay to zero in the free stream. With increasing Prandtl number peak velocity is not only depleted, it is migrated closer to the wall. Both pre- and post-velocity gradients are considerably increased with greater Prandtl number. The higher Prandtl numbers $(10,100)$ are more characteristic of high-density oils encountered in petroleum operations. These naturally attain lower velocities owing to higher viscosities and the wavy surface geometry caan be utilized to achieve acceleration in such fluids imporving efficiency of transport.

Fig. 10 shows the streamwise distribution in Nusselt number (dimensionless heat transfer gradient at the plate surface). Evidently the Nusselt number exhibits maximum values on the crests of the wavy surface while the minimum values occur on the troughs. Highest temperatures are restricted in the troughs since heat is transferred in these zones to fluid essentially by conduction. This results in the lowest values for heat transfer rates at the troughs. The magnitude of peaks grows steadily with distance along the plate i.e. streamwise coordinate. This pattern has also been reported by Yao [5] and many other investigators. It is a classical feature of wavy surface convection flows. The oscillating nature of Nusselt number distribution is clearly captured in fig. 10. Near the plate surface the thermal boundary layer thickness is greater (higher temperatures, lower heat transfer gradients) and further downstream the converse behaviour will occur.

Fig. 11 illustrates the streamwise distribution in surface shear stress function (skin friction at the plate surface). Generally there is a gradual decay in maximum values (peak magnitudes) of skin friction with progression along the plate from the leading edge, downstream. This implies that the flow is steadily decelerated at the plate surface with increasing streamwise coordinate. The oscillatory nature of the decay is clearly visualized in fig. 11. The general trends are also in good agreement with other studies including Yao [5].

\section{CONCLUSIONS}

A mathematical model for the free convection flow of a viscous Newtonian fluid past a wavy surface adjacent to a semi-infinite isotropic non-Darcian porous medium has been presented. This model is a simulation of near-wall flows in petro-chemical transport system. Several special cases of the transformed boundary layer equations have been discussed. A Darcy-Forchheimer drag force model has been utilized to analyze the porous medium impedance effects for highly permeable geomaterials. The transformed nonlinear two-point, boundary value problem has been solved with a pseudo-spectral computational method, combining the Chebyschev spectral collocation technique with the BellmanKalaba quasilinearization method, and termed spectral quasi-linearization method (SQLM). The influence of wave amplitude (geometric parameter), Prandtl number, Forchheimer number, Darcy 
number on the flow characteristics has been evaluated. Validation of the SQLM solutions has been obtained with a two-dimensional smoothed particle hydrodynamics (SPH) code originally developed for solar collectors which adapts well also to petroleum conveying ribbed (wavy geometrical) systems. The engineering parameters of interest, namely the velocity, temperature, Nusselt number and skin friction, have been depicted graphically for the influence of the dominant thermophysical parameters. Temperatures have been shown to be enhanced with increasing wave amplitude and Forchheimer number but with decreasing Darcy number (less permeable regime) and Prandtl number. Velocity is observed to be generally enhanced with wavy amplitude, Darcy number and Forchheimer number but suppressed with increasing Prandtl number. Both Nusselt number and skin friction exhibit a sinusoidal variation with streamwise coordinate and are respectively enhanced and reduced in magnitude with progression along the plate surface downstream from the leading edge. The present study demonstrates the robustness of SQLM computations and is currently being extended to consider more complex nonNewtonian working fluids and viscoelastic flows which are encountered in petroleum operations [5557]. The results of these investigations will be communicated in the near future. Furthermore the present work may also be extended to electrically-conducting fluids in order to address magnetohydrodynamic, slip, dissipative and possibly entropy generation effects, as considered by Adesanya and co-workers [58-62].

\section{FUNDING STATEMENT}

This research received no specific grant from any funding agency in the public, commercial, or notfor-profit sectors.

\section{REFERENCES}

[1] Jie Sun, Jiaqiang Jing, Peiyu Jing, Nian Duan, Cheng Wu, Jiatong Tan, Experimental study on drag reduction of aqueous foam on heavy oil flow boundary layer in an upward vertical pipe, Petroleum Science and Engineering, 146, 409-417 (2016).

[2] L. C. Edomwonyi-Otu, P. Angeli, Pressure drop and holdup predictions in horizontal oil-water flows for curved and wavy interfaces, Chemical Engineering Research and Design, 93, 55-65 (2015).

[3] Y. Peet, P. Sagaut and Y. Charron, Pressure loss reduction in hydrogen pipelines by surface restructuring, Int. J. Hydrogen Energy, 34, 8964-8973 (2009).

[4] Caponi, E.A., Fornberg, B., Knight, D.D., McLean, J.W., Saffman, P.G. and Yuen, H.C., Calculations of laminar viscous flow over a moving wavy surface, J. Fluid Mechanics, 124, 347-362 (1982).

[5] Yao, L.S., Natural convection along a vertical wavy surface, ASME J. Heat Transfer, 105, 465-468 (1983).

[6] Moulic, S.G. and Yao, L.S., Natural convection along a vertical wavy surface with uniform heat flux, ASME J. Heat Transfer, 111, 1106-1108 (1989).

[7] Rees, D.A.S. and Pop, I., Boundary layer flow and heat transfer on a continuous moving wavy surface, Acta Mechanica, 112, 4, 149 - 158 (1995)

[8] Hossain, M. A., Kabir, S. and Rees, D. A. S., Natural convection of fluid with variable viscosity from a heated vertical wavy surface, Zeitshrift fur Angewandte Math. Phys., 53, 48-57 (2002).

[9] Tashtoush, B; Abu-Irshaid, E., Heat and fluid flow from a wavy surface subjected to a variable heat flux, Acta Mechanica, 152, 1-8 (2001). 
[10] Bég, O.Anwar, The Abbasi-Tajik model in thermal convection from wavy surfaces, Technical Report, Aerospace Engineering, Sheffield Hallam University, 94 pages, Sheffield, UK, March (2009).

[11] Hossain, M.Z. and Islam, A.K.M S., Numerical investigation of unsteady flow and heat transfer in wavy channels, $15^{\text {th }}$ Australasian Fluid Mechanics Conference, Sidney, Australia, 13-17 December (2004).

[12] Shalini and Rathish Kumar, B.V., Free convection in a thermally-stratified non-Darcian wavy porous enclosure, J. Porous Media, 4 (2004).

[13] Rahman, S.U. and Badr, H.M., Natural convection from a vertical wavy surface embedded in saturated porous media, Ind. Eng. Chem. Res., 41 (17), 4422 -4429 (2002).

[14] Chiu, C.P. and Chou, H.M., Free convection in the boundary layer flow of a micropolar fluid along a vertical wavy surface, Acta Mechanica, 101, 161-174 (1993).

[15] Varol, Y. and Oztop, HF., Buoyancy induced heat transfer and fluid flow inside a tilted wavy solar collector, Building Environment, 42, 2062-2071 (2007).

[16] K.M. Gawlik and C.F. Kutscher, Wind heat loss from corrugated transpired solar collectors, ASME J Solar Energy Engineering, 124, 256-261 (2002)

[17] Kumar, B.V.R. and Shalini, G., Non-Darcy free convection induced by a vertical wavy surface in a thermally stratified porous medium, Int. J. Heat Mass Transfer. 47,. 2353-2363. (2004).

[18] S.U. Rahman, Natural convection along vertical wavy surfaces: an experimental study, Chem. Eng. J., 84, 587-591 (2001).

[19] S. Ghosh Moulic and L. S. Yao, Mixed convection along a wavy surface, ASME J. Heat Transfer 111(4), 974-979 (1989).

[20] Md Abdul Alim, Md Rezaul Karim, Md Miraj Akand, Heat generation effects on MHD natural convection flow along a vertical wavy surface with variable thermal conductivity, Amer. J. Comp. Mathematics, 2, 42-50 (2012).

[21] R.E. Bellman and R.E. Kalaba, Quasilinearization and Nonlinear Boundary-Value Problems, Elsevier, New York, USA (1965).

[22] L.N. Trefethen, Spectral Methods in MATLAB, SIAM, Philadelphia, USA (2000).

[23] O. Anwar Bég, THERMO-SPH- a smoothed particle simulator for heat transfer from complex geometrical configurations, Technical Report, Gort Engovation, Bradford, UK, THERMO-D-A-154, 49 pages, June (2013).

[24] Coussy, O., Mechanics of Porous Continua, Butterworths, France (1993).

[25] Vafai, K. and Tien, C.L., Boundary and inertial effects on flow and heat transfer in porous media, Int. J. Heat Mass Transfer, 24, 195-203 (1981).

[26] R. G. Owens, C. Chauvière and T. N. Phillips, A locally-upwinded spectral technique (LUST) for viscoelastic flows, J. Non-Newtonian Fluid Mech., 108, 49-72 (2002).

[27] S. Shateyi and S. S. Motsa, Thermal radiation effects on heat and mass transfer over an unsteady stretching surface, Mathematical Problems in Engineering, 2009, 1-13 (2009).

[28] S. Shateyi and S. S. Motsa, Variable viscosity on magnetohydrodynamic fluid flow and heat transfer over an unsteady stretching surface with Hall effect, Bound. Val.Problems, 2010, 1-20 (2010).

[29] P Sibanda, S Motsa, Z Makukula, A spectral-homotopy analysis method for heat transfer flow of a third grade fluid between parallel plates, International Journal Numerical Methods Heat Fluid Flow 22 (1), 4-23 (2011).

[30] O. Anwar Bég, M. Hameed and Tasveer A. Bég, Chebyshev spectral collocation simulation of nonlinear boundary value problems in electrohydrodynamics (EHD), Int. J. Computational Methods in Engineering Science and Mechanics, 14 (2) 104-115 (2013).

[31] Md Mainul Hoque; Md Mahmud Alam; M Ferdows, O. Anwar Bég, Numerical simulation of Dean number and curvature effects on magneto-biofluid flow through a curved conduit, Proc. IMECHE- Part H; J. Engineering in Medicine, 227 (11):1155-70 (2013).

[32] C. Liang, J. Chen and J. D. Lee, Spectral difference solution of two-dimensional unsteady compressible micropolar equations on moving and deformable grids, $50^{\text {th }}$ AIAA Aerospace Sciences Meeting, 9-12 January, Nashville, Tennessee, USA (2012).

[33] O. Anwar Bég, S. S. Motsa, Ali Kadir, T. A. Bég, M.N. Islam, Spectral quasilinear numerical simulation of micropolar convective wall plumes in high permeability porous media, J. Engineering Thermophyiscs (2016). Submitted. 
[34] O. Anwar Bég, Sandile S. Motsa, M.N. Islam, M.D. Lockwood, Pseudo-spectral and variational iteration simulation of exothermically-reacting Rivlin-Ericksen viscoelastic flow and heat transfer in a rocket propulsion duct, Comp. Thermal Sciences, 6 (2): 91-102 (2014).

[35] Gottlieb, D and Orszag, S.A., Numerical analysis of spectral methods, Society for Industrial and Applied Mathematics (SIAM), Philadelphia, USA (1977).

[36] W. S. Don and A. Solomonoff, Accuracy and speed in computing the Chebyshev collocation derivative, SIAM Journal on Scientific Computing, 16, 1253-1268 (1995).

[37] R.E. Bellman and R.E. Kalaba, Quasilinearization and Nonlinear Boundary-Value Problems, Elsevier, New York, USA (1965).

[38] C. Canuto, M. Hussaini, A. Quarteroni, and T. Zang, Spectral Methods in Fluid Dynamics, Springer, Berlin (1993).

[39] Lucy L.B., A numerical approach to the testing of the fission hypothesis, Astro. J., 82, 1013-1024 (1977).

[40] J. J. Monaghan, R. A. Gingold, Shock simulation by the particle method SPH, J. Computational Physics, 52, 374-389 (1983).

[41] J. J. Monaghan, Smoothed particle hydrodynamics, Ann. Rev. Astro. Astro., 30, 543-574 (1992).

[42] J. J. Monaghan, Simulating free surface flows with SPH, J. Computational Physics, 110, 399-406 (1994).

[43] J. J. Monaghan, SPH without a tensile instability, J. Comp. Physics, 159, 290-311 (2000).

[44] J. P. Morris, P. J. Fox, Y. Zhu, Modeling low Reynolds number incompressible flows using SPH, J. Computational Physics, 136, 214-226 (1997).

[45] G. Oger, M. Doring, B. Alessandrini, P. Ferrant, Two-dimensional SPH simulations of wedge water entries, J. Computational Physics, 213, 803-822 (2006).

[46] S. Comas-Cardona, P. H. L. Groenenboom, C. Binetruy, P. Krawczak, A generic mixed FE-SPH method to address hydro-mechanical coupling in liquid composite moulding processes, Composites: Part A, 36, 1004-1 010 (2005).

[47] L. D. G. Sigalotti, J. Klapp, E. Sira, Y. Melean, and A. Hasmy, SPH simulations of timedependent Poiseuille flow at low Reynolds numbers, J. Comput. Phys., 191, 622-638 (2003).

[48] Y. Zhu and P. J. Fox, Smoothed particle hydrodynamics model for diffusion through porous media, Transport Porous Media, 43, 441-471 (2001).

[49] P. W. Cleary and J. J. Monaghan, Conduction modelling using smoothed particle hydrodynamics, J. Comp. Phys., 148, 235-236 (1999).

[50] O. Anwar Bég, SPLASH- A smoothed particle hydrodynamic solver for spacecraft "splashdown" simulations in MATLAB, Technical Report for European Space Consortium- AERO-D-61-SPH, Gort Engovation- Engineering Sciences, Bradford, UK, 85 pages, July (2013).

[51] O. Anwar Bég and Tasveer A. Bég, Smoothed particle hydrodynamic simulation of magnetic field materials processing, Technical Report for Metallurgy Industry (Norway)- MANUFACT-M-14SPH, Gort Engovation- Engineering Sciences, Bradford, UK, 64 pages, August (2013).

[52] G. R. Liu and M. B. Liu, Smoothed Particle Hydrodynamics: A Meshfree Particle Method, World Scientific Publishing Co., Singapore (2003).

[53] O. Anwar Bég, WAVY-SPH: A smoothed particle hydrodynamic solver for wavy solar collectors, Technical Report- SOLAR-E-SPH, Gort Engovation- Bradford, UK, 85 pp, August (2013).

[54] M. S. Abdallah and B. Zeghmati, Effects of the wavy surface on free convection-radiation along an inclined plate, WASET Journal, 78, 458-464 (2013).

[55] V. R. Prasad, S. A. Gaffar and O. Anwar Bég, Heat and mass transfer of a nanofluid from a horizontal cylinder to a micropolar fluid, AIAA J. Thermophysics Heat Transfer, 29, 127-139 (2015).

[56] M.J. Uddin, O. Anwar Bég and N.S. Amin, Hydromagnetic transport phenomena from a stretching or shrinking nonlinear nanomaterial sheet with Navier slip and convective heating: a model for bio-nano-materials processing, J. Magnetism Magnetic Materials, 368, 252-261(2014).

[57] TB Chang, A Mehmood, O. Anwar Bég, M Narahari, MN Islam, F Ameen, Numerical study of transient free convective mass transfer in a Walters-B viscoelastic flow with wall suction, Comm. Nonlinear Science and Numerical Simulation, 16, 216-225 (2011).

[58] S. O. Adesanya and O. D. Makinde, MHD oscillatory slip flow and heat transfer in a channel filled with porous media, U.P.B Scientific Bulletin A, 76, pp197- 204 (2014). 
[59] S. O. Adesanya, J.A. Falade and O. D. Makinde, Pulsating flow through vertical porous channel with viscous dissipation effect, U.P.B. Sci. Bull., Series D - Mechanical Engineering vol. 77(1), pp 25-36 (2015).

[60] S. O. Adesanya, J.A. Falade, Thermodynamic analysis of hydromagnetic third grade fluid flow through a channel filled with porous medium, Alexandria Engineering Journal, 54, 615-622 (2015).

[61] S. O. Adesanya, E.O. Oluwadare, J.A. Falade and O.D. Makinde, Hydromagnetic natural convection flow between vertical parallel plates with time-periodic boundary conditions, Journal of Magnetism and Magnetic Materials, 396, 295-303 (2015).

[62] S. O. Adesanya, S.O. Kareem, JA. Falade and S.A. Arekete, Entropy generation analysis for a reactive couple stress fluid flow through a channel saturated with porous material, Energy, 93, 1239 - 1245 (2015). 
TABLES

\begin{tabular}{|c|c|c|c|c|}
\hline & $\begin{array}{c}F^{\prime \prime}(0) \\
\text { (skin friction) }\end{array}$ & $\begin{array}{c}F^{\prime \prime}(0) \\
\text { (skin friction) }\end{array}$ & $\begin{array}{c}-\theta^{\prime}(0) \\
\text { (wall heat } \\
\text { transfer rate) } \\
\text { SQLM }\end{array}$ & $\begin{array}{c}-\theta^{\prime}(0) \\
\text { (wall heat } \\
\text { transfer rate) } \\
\text { SPH }\end{array}$ \\
\hline$x$ & SQLM & SPH & 1.16255 & 1.16256 \\
\hline 0.0 & 0.41700 & 0.41701 & 0.61923 & 0.61921 \\
\hline 0.5 & 0.14470 & 0.14471 & 0.58951 & 0.58950 \\
\hline 1.0 & 0.12276 & 0.12274 & 0.57828 & 0.57826 \\
\hline 1.5 & 0.11996 & 0.11994 & 0.57274 & 0.57273 \\
\hline 2.0 & 0.11903 & 0.11902 & 0.56838 & 0.56837 \\
\hline 2.5 & 0.11817 & 0.11818 & 0.56433 & 0.56432 \\
\hline 3.0 & 0.11734 & 0.11733 & 0.56049 & 0.56047 \\
\hline 3.5 & 0.11655 & 0.11654 & 0.55683 & 0.55682 \\
\hline 4.0 & 0.11579 & 0.11580 & \\
\hline
\end{tabular}

Table 1: SQLM and SPH comparative computations for skin friction (surface shear stress function) with $F s=1, D a=1, \operatorname{Re}=10, a=0.2, \operatorname{Pr}=10$.

\begin{tabular}{|c|c|c|c|c|}
\hline & $\begin{array}{c}F^{\prime \prime}(0) \\
\text { (skin friction) }\end{array}$ & $\begin{array}{c}F^{\prime \prime}(0) \\
\text { (skin friction) }\end{array}$ & $\begin{array}{c}-\theta^{\prime}(0) \\
\text { (wall heat } \\
\text { transfer rate) } \\
\text { SQLM }\end{array}$ & $\begin{array}{c}-\theta^{\prime}(0) \\
\text { (wall heat } \\
\text { transfer rate) } \\
\text { SPH }\end{array}$ \\
\hline $\mathrm{X}$ & $\mathbf{S Q L M}$ & $\mathbf{S P H}$ & 1.16255 & 1.16254 \\
\hline 0.0 & 0.41700 & 0.41701 & 0.53261 & 0.53262 \\
\hline 0.5 & 0.10846 & 0.10845 & 0.50470 & 0.50471 \\
\hline 1.0 & 0.10289 & 0.10290 & 0.48516 & 0.48514 \\
\hline 2.5 & 0.09897 & 0.09895 & 0.46975 & 0.46973 \\
\hline 2.0 & 0.09591 & 0.09593 & 0.45694 & 0.45693 \\
\hline 3.0 & 0.09337 & 0.09338 & 0.44597 & 0.44598 \\
\hline 3.5 & 0.09121 & 0.09123 & 0.43637 & 0.43638 \\
\hline 4.0 & 0.08932 & 0.08931 & 0.42783 & 0.42782 \\
\hline
\end{tabular}

Table 2: SQLM and SPH comparative computations for skin friction (surface shear stress function) with $F s=1, D a=0.1, \operatorname{Re}=10, a=0.2, \operatorname{Pr}=10$.

\begin{tabular}{|c|c|c|c|c|}
\hline$x$ & $\begin{array}{c}F^{\prime \prime}(0) \\
\text { (skin friction) } \\
\text { SQLM }\end{array}$ & $\begin{array}{c}F^{\prime \prime}(0) \\
\text { (skin friction) } \\
\text { SPH }\end{array}$ & $\begin{array}{c}-\theta^{\prime}(0) \\
\text { (wall heat } \\
\text { transfer rate) } \\
\text { SQLM }\end{array}$ & $\begin{array}{c}-\theta^{\prime}(0) \\
\text { (wall heat } \\
\text { transfer rate) } \\
\text { SPH }\end{array}$ \\
\hline 0.0 & 0.41700 & 0.41702 & 1.16255 & 1.16256 \\
\hline 0.5 & 0.11549 & 0.11548 & 0.56050 & 0.56049 \\
\hline 1.0 & 0.11048 & 0.11047 & 0.53515 & 0.53514 \\
\hline 1.5 & 0.10671 & 0.10672 & 0.51648 & 0.51647 \\
\hline 2.0 & 0.10367 & 0.10366 & 0.50139 & 0.50138 \\
\hline 2.5 & 0.10112 & 0.10113 & 0.48868 & 0.48867 \\
\hline 3.0 & 0.09891 & 0.09892 & 0.47766 & 0.47765 \\
\hline 3.5 & 0.09697 & 0.09698 & 0.46795 & 0.46794 \\
\hline 4.0 & 0.09523 & 0.09522 & 0.45926 & 0.45927 \\
\hline
\end{tabular}

Table 3: SQLM and SPH comparative computations for skin friction (surface shear stress function) with $F s=10, D a=1, \operatorname{Re}=10, a=0.2, \operatorname{Pr}=10$. 


\section{FIGURES}

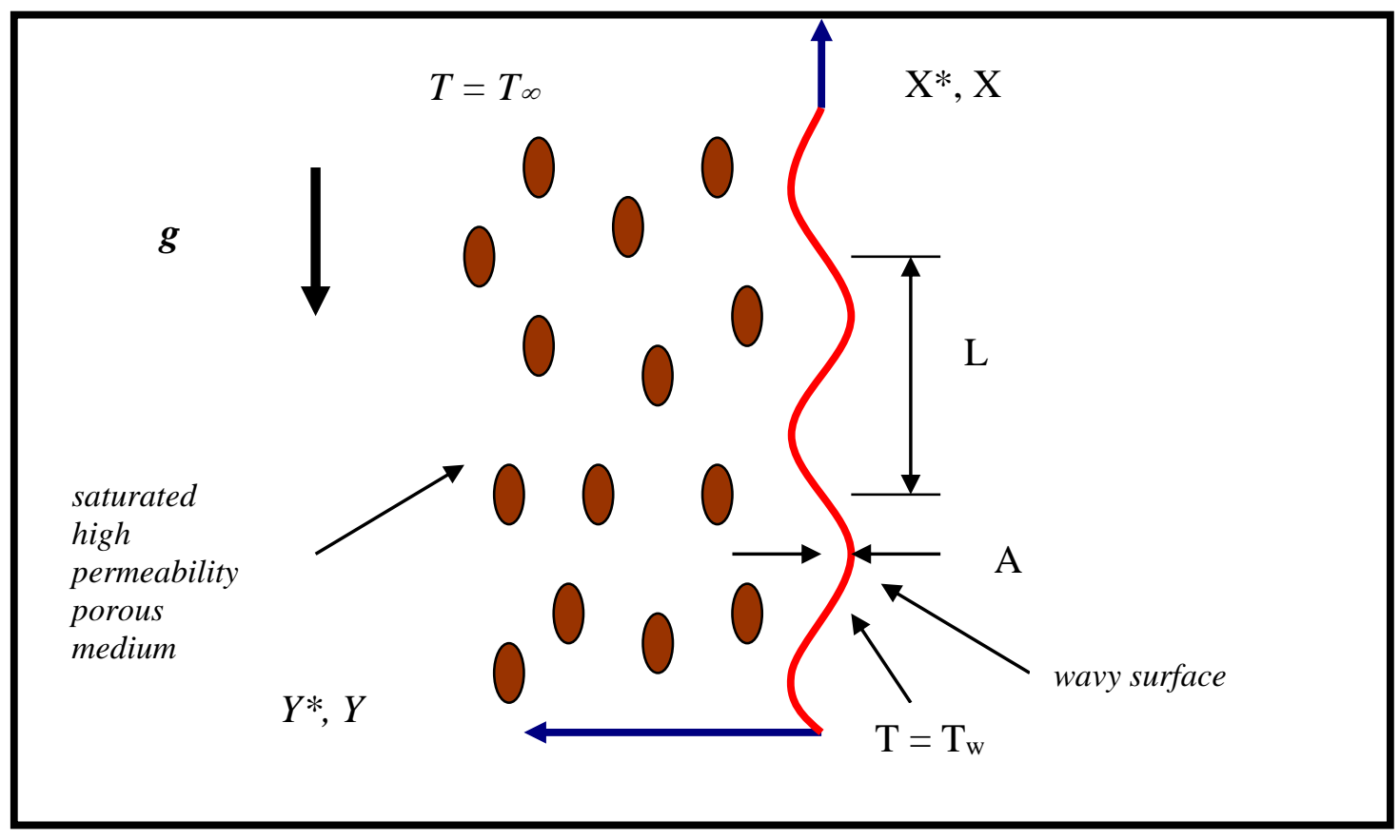

Figure 1: Geometry of the two-dimensional flow regime

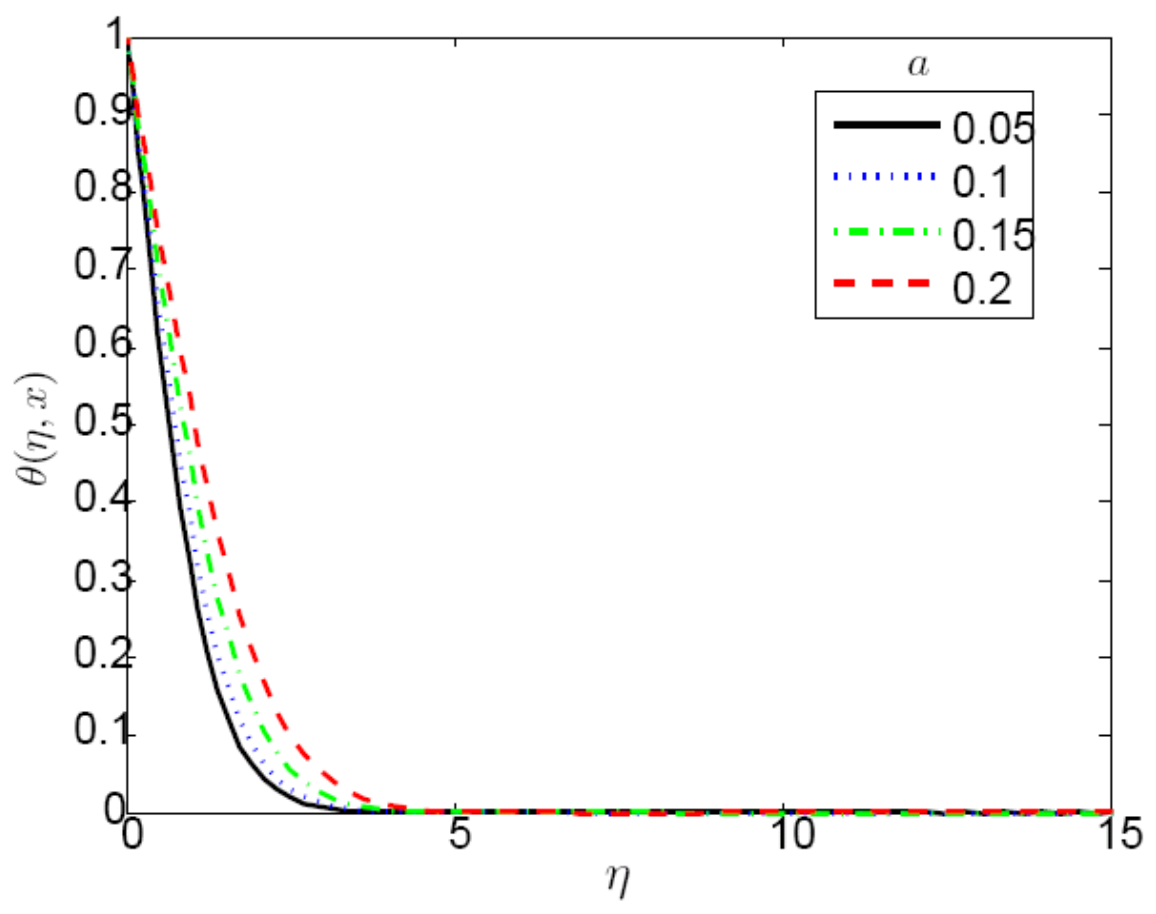

Fig. 2: Temperature distribution with transverse coordinate for $F s=1, D a=1, \operatorname{Re}=10, \operatorname{Pr}=10$ at $x$ = 1 for various amplitude parameters. 


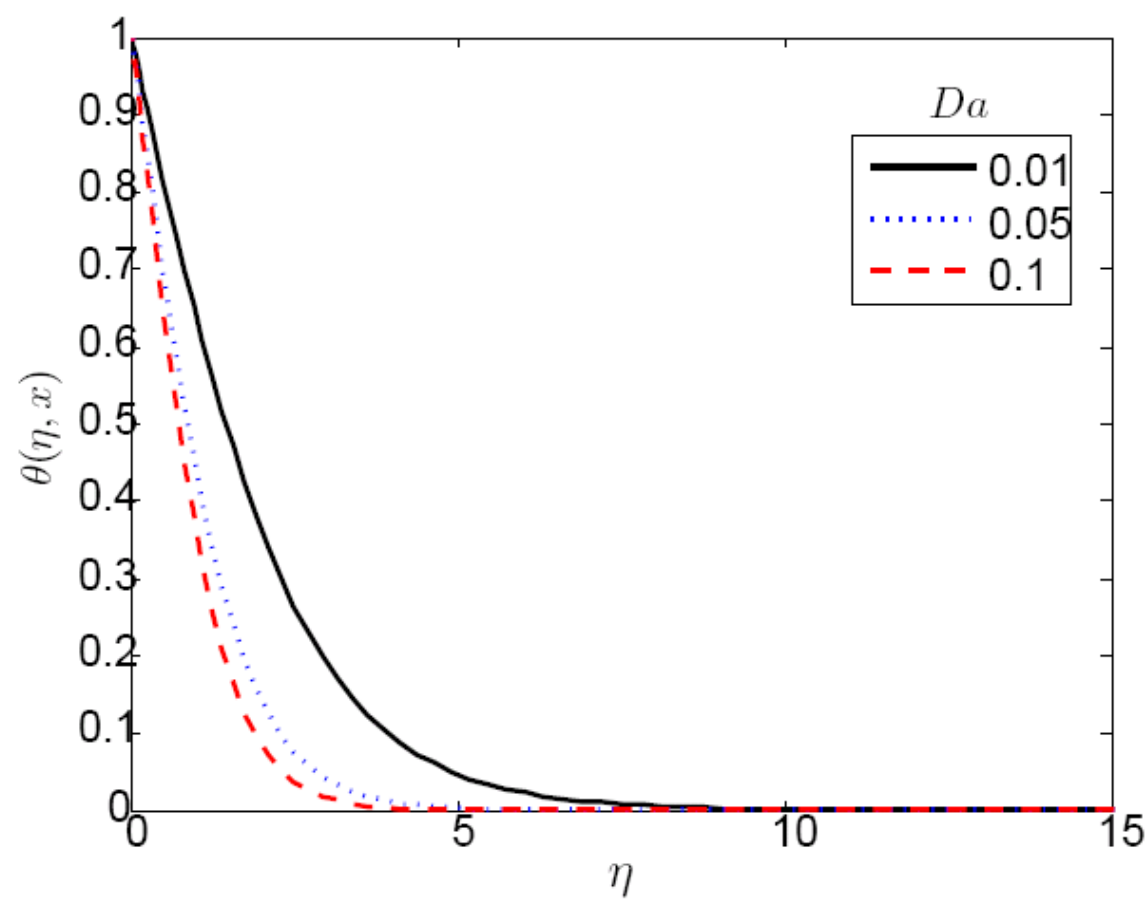

Fig. 3: Temperature distribution with transverse coordinate for $F s=1, a=0.2, \operatorname{Re}=10, \operatorname{Pr}=10$ at $x$ $=1$, for various Darcy numbers.

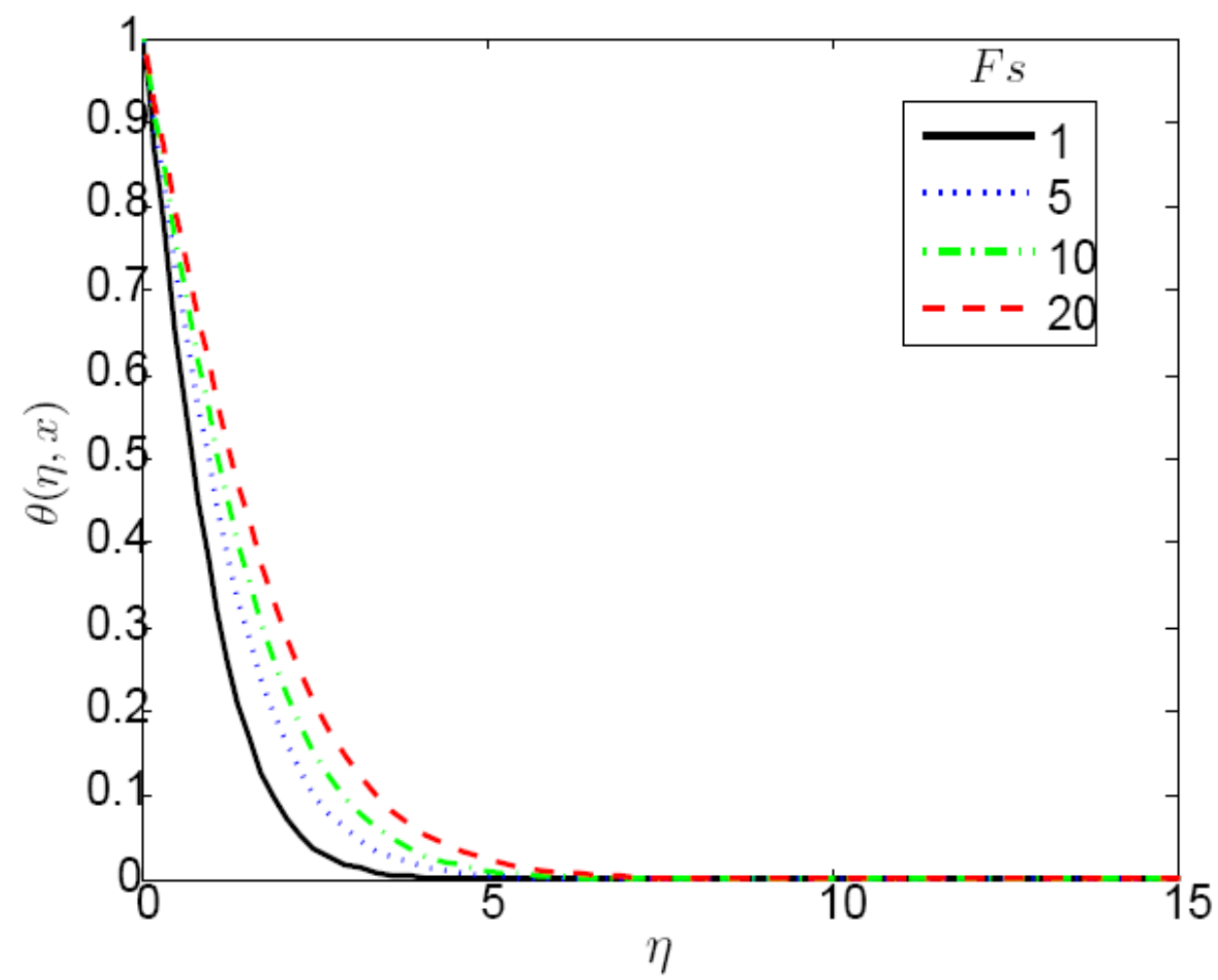

Fig. 4: Temperature distribution with transverse coordinate for $D a=1, a=0.2, \operatorname{Re}=10, \operatorname{Pr}=10$ at $x$ $=1$ for various Forchheimer numbers. 


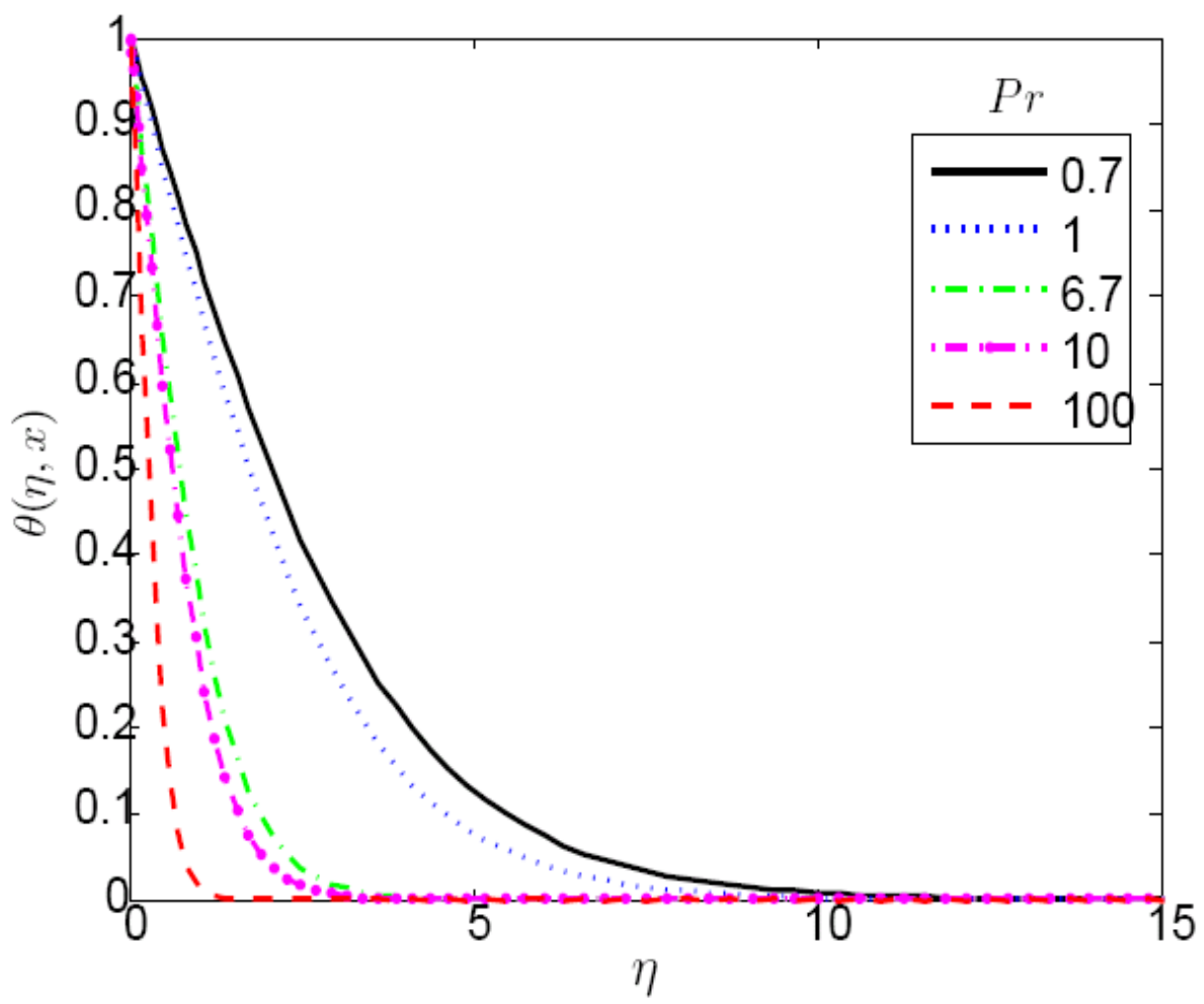

Fig. 5: Temperature distribution with transverse coordinate for $D a=1, F s=1, a=0.2, \operatorname{Re}=10$ at $x$ 1 for various Prandtl numbers.

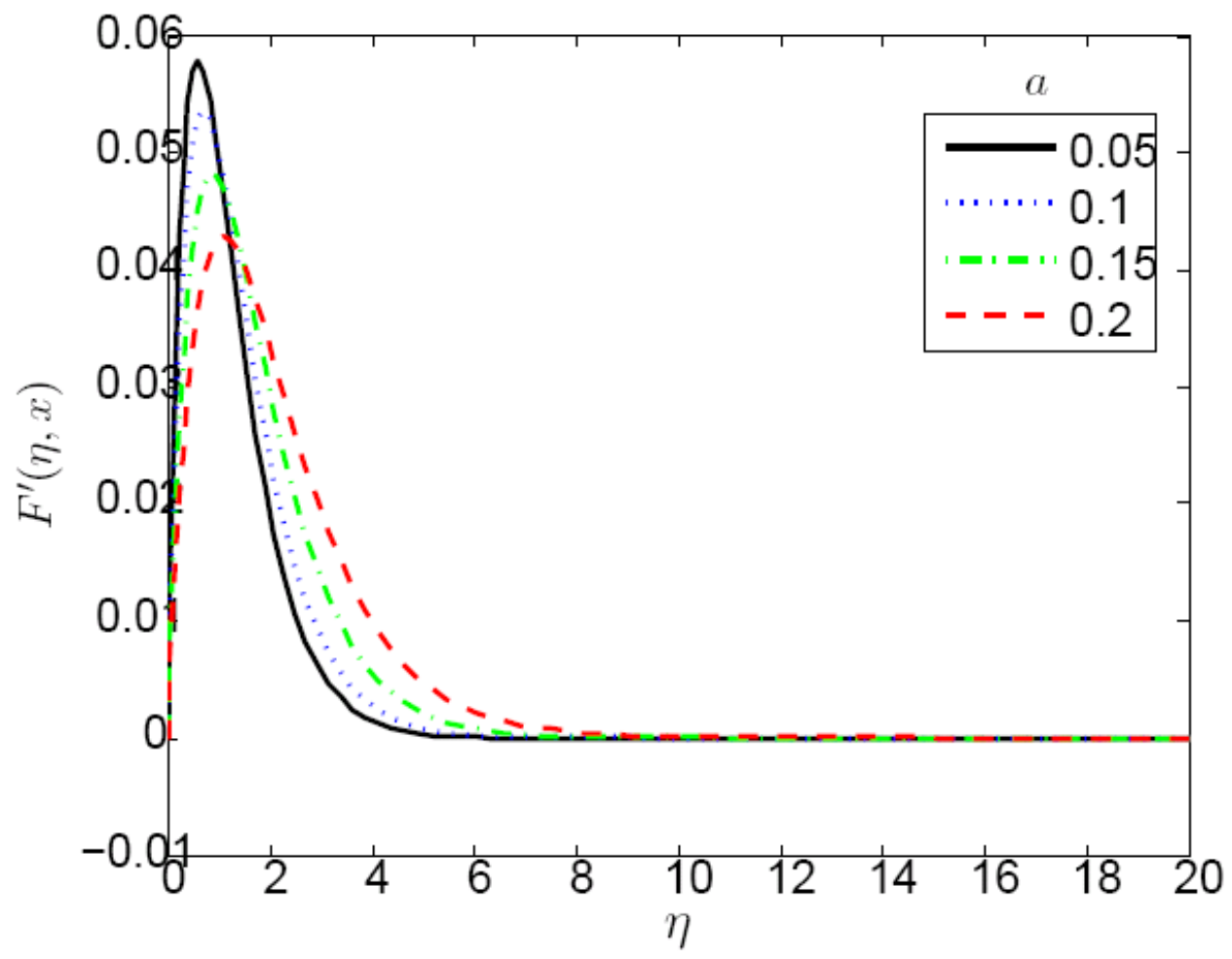

Fig. 6: Velocity distribution with transverse coordinate for $F s=1, D a=1, \operatorname{Re}=10, \operatorname{Pr}=10$ at $x=1$, for various wave amplitude numbers. 


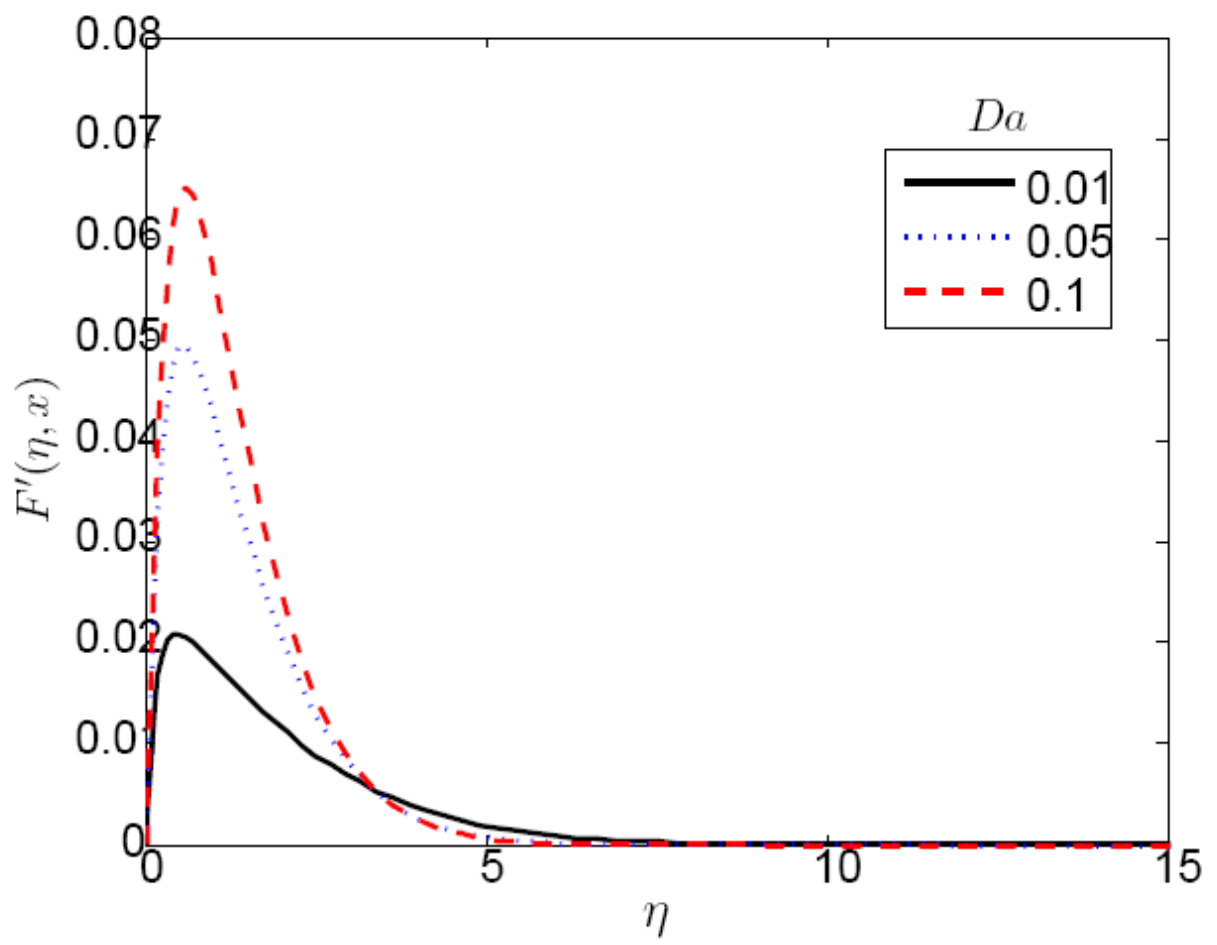

Fig. 7: Velocity distribution with transverse coordinate for $F s=1, a=0.2, \operatorname{Re}=10, \operatorname{Pr}=10$ at $x=1$, for various Darcy numbers.

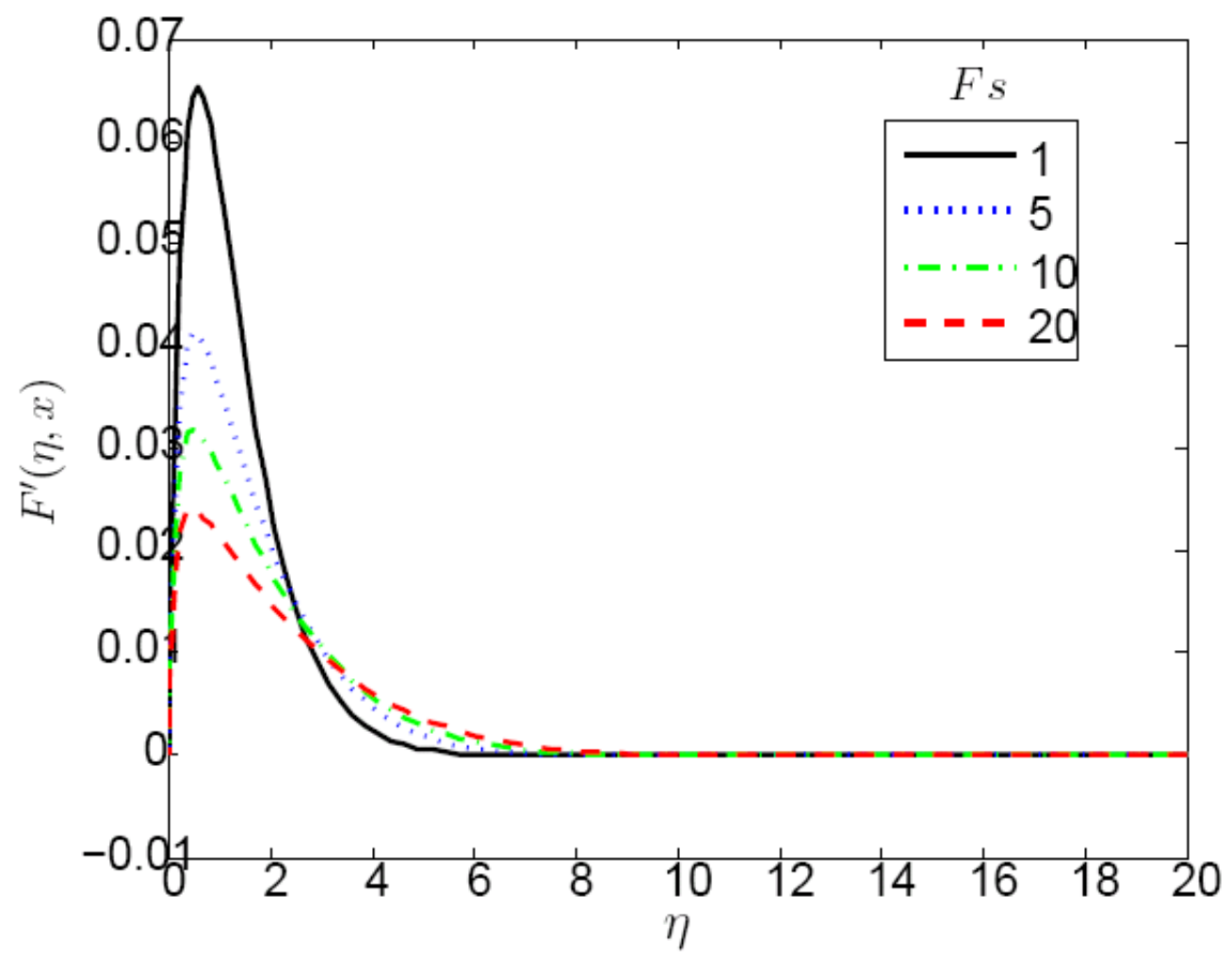

Fig. 8: Velocity distribution with transverse coordinate for $D a=1, a=0.2, \operatorname{Re}=10, \operatorname{Pr}=10$ at $x=1$, for various Forchheimer numbers. 


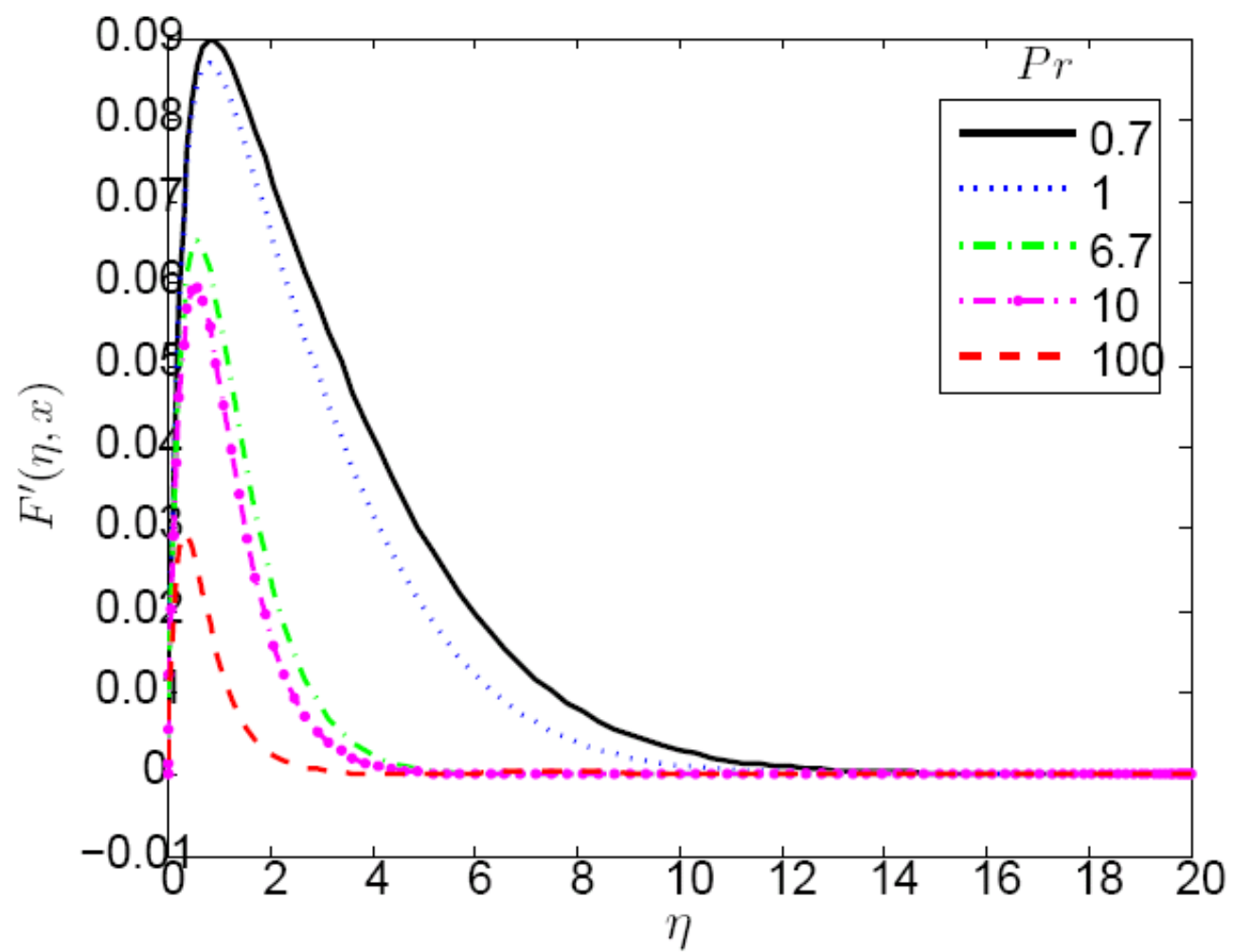

Fig. 9: Velocity distribution with transverse coordinate for $D a=1, a=0.2, \operatorname{Re}=10, F s=1$, at $x=1$, for various Prandtl numbers.

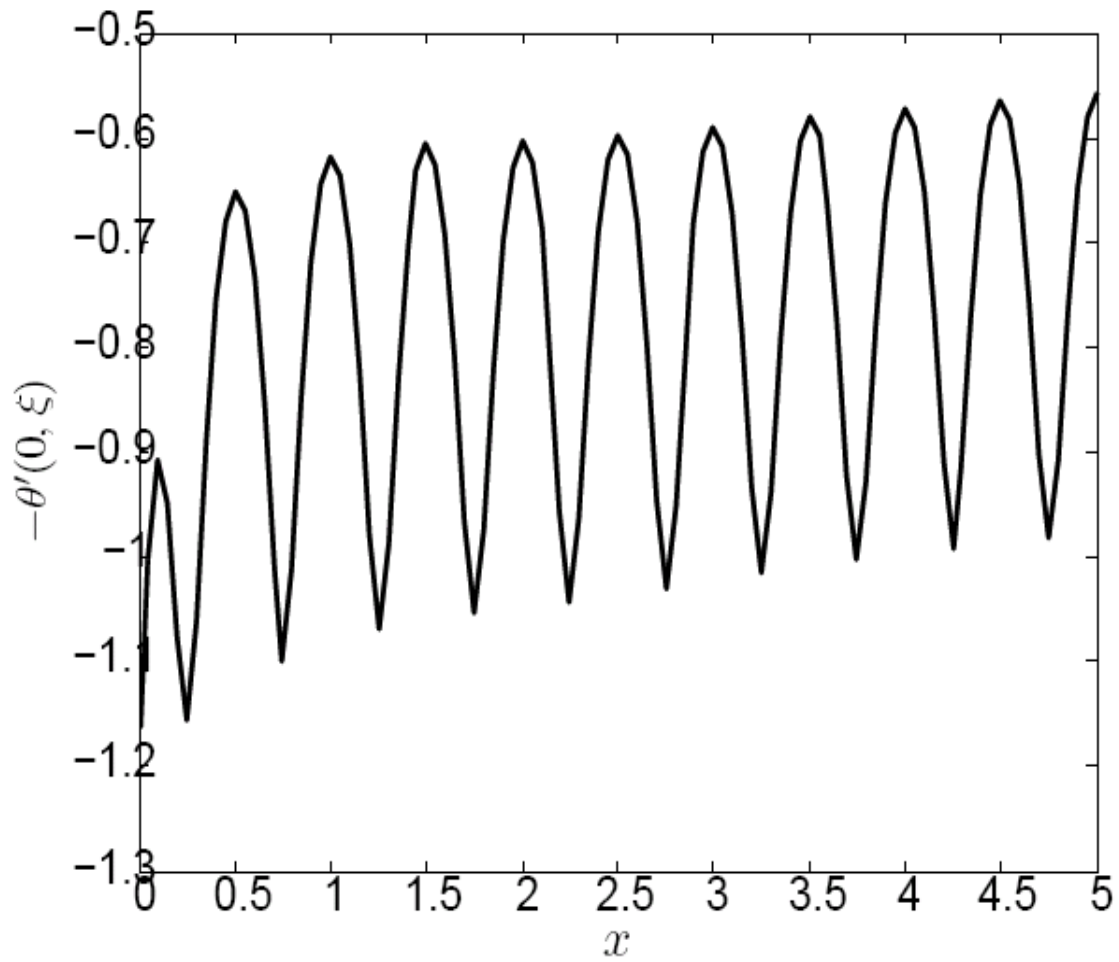

Fig. 10: Nusselt number (dimensionless heat transfer gradient) distribution with streamwise coordinate for $D a=1, a=0.2, \operatorname{Re}=10, F s=1, \operatorname{Pr}=10$. 


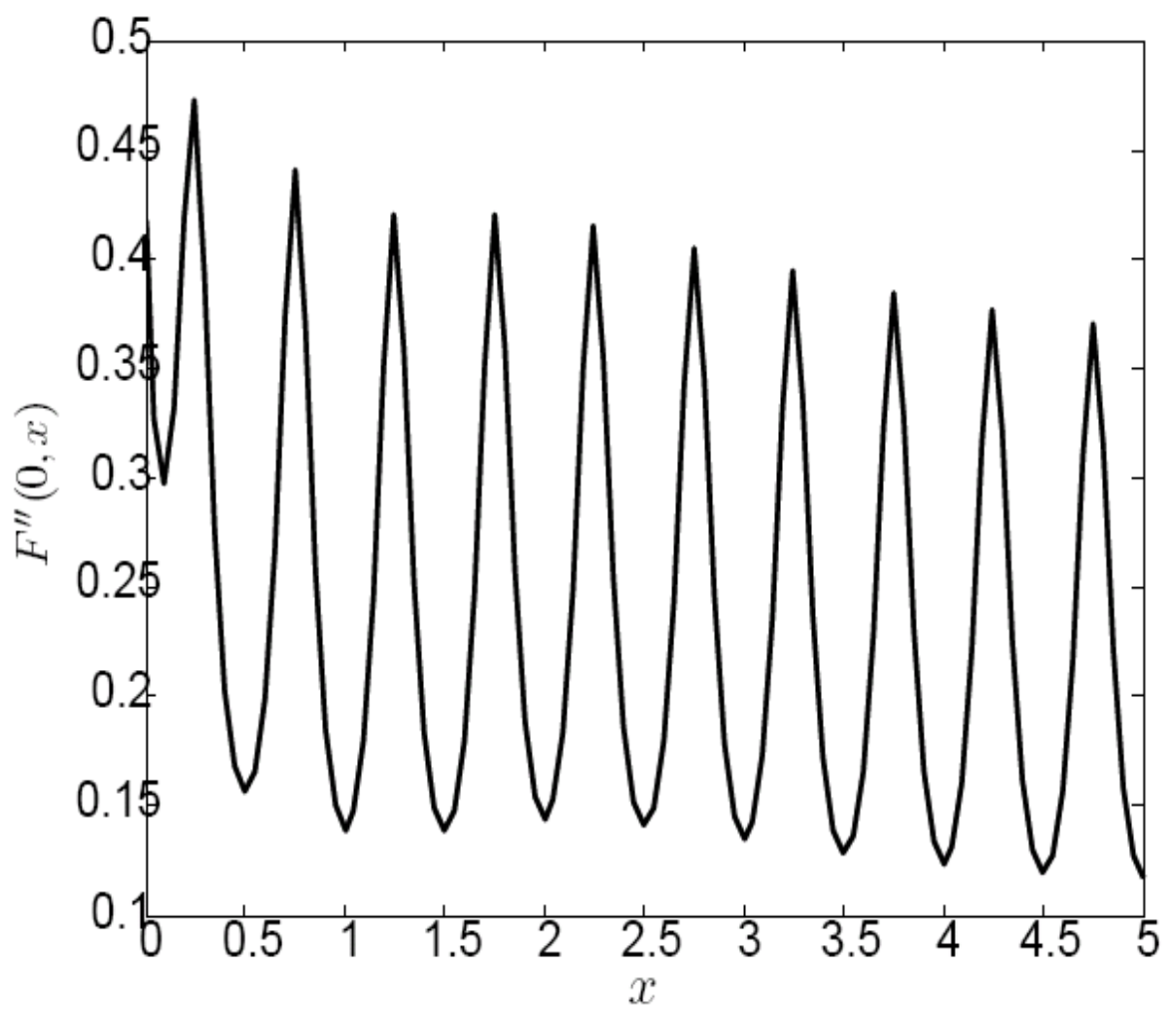

Fig. 11: Skin friction (dimensionless surface shear stress) distribution with streamwise coordinate for $D a=1, a=0.2, \operatorname{Re}=10, F s=1, \operatorname{Pr}=10$. 